\title{
Intrauterine Growth Restriction Followed by Oxygen Support Uniquely Interferes with Genetic Regulators of Myelination
}

\author{
Dill Chang, ${ }^{1}$ Abhineet Sharma, ${ }^{1}$ Mirrah Bashir, ${ }^{1}$ Camille M. Fung, ${ }^{2}$ Robert W. Dettman, ${ }^{1}$ and \\ Maria L. V. Dizon ${ }^{1}$
}

https://doi.org/10.1523/ENEURO.0263-20.2021

${ }^{1}$ Department of Pediatrics, Division of Neonatology, Ann \& Robert H. Lurie Children's Hospital of Chicago, Chicago, IL 60611 and ${ }^{2}$ Department of Pediatrics, University of Utah, Salt Lake City, UT 84112

\begin{abstract}
Intrauterine growth restriction (IUGR) and oxygen exposure in isolation and combination adversely affect the developing brain, putting infants at risk for neurodevelopmental disability including cerebral palsy (CP). Rodent models of IUGR and postnatal hyperoxia have demonstrated oligodendroglial (OL) injury with subsequent white matter injury (WMI) and motor dysfunction. Here, we investigate transcriptomic dysregulation in IUGR with and without hyperoxia exposure to account for the abnormal brain structure and function previously documented. We performed RNA sequencing and analysis using a mouse model of IUGR and found that IUGR, hyperoxia, and the combination of IUGR with hyperoxia (IUGR/hyperoxia) produced distinct changes in gene expression. IUGR in isolation demonstrated the fewest differentially expressed genes (DEGs) compared with control. In contrast, we detected several gene alterations in IUGR/hyperoxia; genes involved in myelination were strikingly downregulated. We also identified changes to specific regulators including TCF7L2, BDNF, SOX2, and DGCR8, through ingenuity pathway analysis (IPA), that may contribute to impaired myelination in IUGR/hyperoxia. Our findings show that IUGR with hyperoxia induces unique transcriptional changes in the developing brain. These indicate mechanisms for increased risk for WMI in IUGR infants exposed to oxygen and suggest potential therapeutic targets to improve motor outcomes.
\end{abstract}

Key words: cerebral palsy; oligodendroglial; perinatal brain injury; RNA sequencing; white matter injury

\section{Significance Statement}

This study demonstrates that perinatal exposures of intrauterine growth restriction (IUGR) and/or postnatal hyperoxia result in distinct transcriptomic changes in the developing brain. In particular, we found that genes involved in normal developmental myelination, myelin maintenance, and remyelination were most dysregulated when IUGR was combined with hyperoxia. Understanding how multiple risk factors lead to white matter injury (WMI) is the first step in developing future therapeutic interventions. Additionally, because oxygen exposure is often unavoidable after birth, an understanding of gene perturbations in this setting will increase our awareness of the need for tight control of oxygen use to minimize future motor disability.

\section{Introduction}

White matter injury (WMI) following in utero hypoxic-ischemic $(\mathrm{HI})$ events, stroke and prematurity is well

Received June 12, 2020; accepted April 23, 2021; First published June 7, 2021.

The authors declare no competing financial interests. documented (Volpe et al., 2011; Back, 2015; van Tilborg et al., 2018). The WMI that occurs secondary to these disease processes puts neonates at greater risk of developing

Author contributions: J.C., R.W.D., and M.L.V.D. designed research; J.C. and M.B. performed research; J.C., A.S., R.W.D., and M.L.V.D. analyzed data; J.C., C.M.F., R.W.D., and M.L.V.D. wrote the paper. 
motor dysfunction including cerebral palsy (CP; Silbereis et al., 2010). There remains, however, a large percentage of neonates that develop CP who have no history of one of these identified perinatal incidents. This gap in knowledge makes it difficult to develop therapeutic interventions for what often results in lifelong disability.

Intrauterine growth restriction (IUGR) is defined as a significant reduction in fetal growth resulting in birth weight $<10$ th percentile for gestational age (Battaglia and Lubchenco, 1967; Schroder, 2003). It affects $\sim 5 \%$ of pregnancies worldwide (Mandruzzato et al., 2008; Tolcos et al., 2011) and results in an increased risk of mortality and significant morbidities. A number of population-based cohort studies have shown a 5- to 7-fold increased risk of developing $\mathrm{CP}$ in growth restricted infants (Ahlin et al., 2013; Dahlseng et al., 2014; Blair and Nelson, 2015; Freire et al., 2015; Streja et al., 2015; Mor et al., 2016). Existing animal studies of IUGR demonstrate evidence of oligodendroglial (OL) injury and subsequent WMI (Olivier et al., 2007; Tolcos et al., 2011; Volpe et al., 2011; Reid et al., 2012; Basilious et al., 2015; Rideau Batista Novais et al., 2016), specifically, a decrease in immature/premyelinating OLs and total OL population (Chang et al., 2018). This is similar to findings in models of prematurity and $\mathrm{HI}$ injury where blocked maturation of oligodendrocyte progenitor cells (OPCs) is implicated in impaired white matter development (Chang et al., 2018). Despite these cellular changes, the molecular mechanisms remain incompletely understood. Alterations in gene expression and signaling pathways that lead to abnormal white matter development in the developing brain are under investigation (Hedtjarn et al., 2004; Guedj et al., 2015; Rideau Batista Novais et al., 2016).

Interestingly, not all IUGR infants go on to develop CP (Ahlin et al., 2013; Dahlseng et al., 2014; Blair and Nelson, 2015; Freire et al., 2015; Streja et al., 2015; Mor et al., 2016), suggesting that additional unidentified factors are involved in the WMI that occurs in this population. It is well documented that, in addition to neurodevelopmental impairment, growth restricted infants are at an increased risk of cardiovascular and pulmonary morbidities, including bronchopulmonary dysplasia and pulmonary hypertension (Rozance et al., 2011; Eriksson et al., 2014; Mestan et al., 2014). To provide appropriate support of their cardiorespiratory status, these infants are often

This work was supported by Illumina NUSeq Pilot Program Grant (J.C.), Ann \& Robert H. Lurie Children's Hospital of Chicago Pediatric Physician-Scientist Research Award (J.C.), the NCATS NIH HHS Grant TL1TR001423 (to A.S.), the NINDS Grant R01NS086945 (to M.L.V.D.), and the NICHD Grant K08 HD102023 (to J.C.).

Acknowledgements: We thank NUSeq and the Advanced Bioinformatics and Bio-Computation Core Facility at the Center for Genetic Medicine at Northwestern University.

Correspondence should be addressed to Jill Chang at jill.chang@ northwestern.edu.

https://doi.org/10.1523/ENEURO.0263-20.2021

Copyright (C) 2021 Chang et al.

This is an open-access article distributed under the terms of the Creative Commons Attribution 4.0 International license, which permits unrestricted use, distribution and reproduction in any medium provided that the original work is properly attributed. admitted to the neonatal intensive care unit and exposed to supraphysiologic oxygen. Rodent studies have found that hyperoxia exposure alone results in damage to the developing white matter including ultrastructural changes in myelin, decreased total OLs, and decreased myelin proteins (Gerstner et al., 2008; Schmitz et al., 2011; Ramani et al., 2013; Ritter et al., 2013). Perinatal brain injury is likely the result of multiple exposures during a critical neurodevelopmental window (Schmitz et al., 2011; Basilious et al., 2015). A multihit hypothesis would provide an explanation for why some IUGR infants go on to develop more severe motor dysfunction and CP. Understanding how multiple risk factors affect the developing brain leading to WMI can lead to modifiable clinical approaches in this highrisk population.

Recently, the multihit hypothesis was tested in a mouse model of IUGR, with and without supplemental oxygen (Chang et al., 2018). Postnatal hyperoxia exposure independently resulted in white matter dysfunction different from that seen in IUGR. IUGR demonstrated changes in OL populations and myelin thickness, while hyperoxia resulted in impaired myelin integrity and decreased white matter tract volume on MRI, suggesting a different mechanism of injury between these exposures. Additionally, a persistent and more complex type of WMI was seen with the combined insult of IUGR with hyperoxia. In contrast to transient changes in OL populations in IUGR, IUGR with hyperoxia resulted in sustained OL alterations into adulthood. The combination of IUGR and hyperoxia also led to more pronounced WMI on MRI and gait changes in adult mice (Chang et al., 2018). The different findings seen with these perinatal exposures support a multihit hypothesis for WMI and also highlight that different mechanisms may be involved with each exposure.

Here, we evaluated the multihit injury model using RNA sequencing (RNA-seq), to test the hypothesis that IUGR and postnatal hyperoxia alter distinct gene networks involved in brain development. We found that IUGR and hyperoxia alone did, indeed, result in distinct changes in gene expression. We also observed that the combination of IUGR with hyperoxia (IUGR/hyperoxia), compared with either condition in isolation, specifically affected genes related to myelination. ingenuity pathway analysis (IPA) identified significant dysregulation of the $\mathrm{Wnt} / \beta$-catenin signaling pathway through TCF7L2 in both hyperoxia and IUGR/hyperoxia. Additionally, IPA identified dysregulation of specific regulators BDNF, SOX2, and DGCR8 that may be responsible for the impaired myelination in IUGR/hyperoxia. These findings demonstrate that different perinatal exposures result in distinct transcriptomic changes in the developing brain, and further support our multihit hypothesis that exposure of growth restricted infants to therapeutic oxygen results in WMI and potential development of CP.

\section{Materials and Methods}

\section{Animals}

Wild-type C57BL/6 mice were purchased from Charles River. All mice were housed in a facility with a 12/12 $h$ 
light/dark cycle and allowed access to food and water ad libitum. Experiments were conducted according to protocols approved by the Institutional Animal Care and Use Committee and Northwestern Center for Comparative Medicine. Animal procedures were conducted in accordance with the National Institutes of Health Guide for the Care and Use of Laboratory Animals.

\section{Murine IUGR model}

Uteroplacental insufficiency is the most common cause of IUGR in developed countries (Fung et al., 2011). Thromboxane $A_{2}\left(T X A_{2}\right)$, is a vasoconstrictor overly expressed in mothers whose pregnancies are complicated by hypertension, cigarette smoking, and poorly controlled diabetes (McAdam et al., 2005; Hayakawa et al., 2006; Fung et al., 2011; Gibbins et al., 2018). Infusion of $\mathrm{TXA}_{2}-$ analog U-46619 has been demonstrated to result in placental vasculature reduction, suggesting placental vascular insufficiency, similar to human placental pathology resulting in IUGR (Gibbins et al., 2018). This model does not require invasive surgery, and it is physiologically relevant to human IUGR pregnancies.

Micro-osmotic Alzet pumps (model 1007D, $0.5 \mathrm{ml} / \mathrm{h}$; DURECT Corporation) were implanted into gravid mice at $12.5 \mathrm{~d}$ after coitus, correlating with the last trimester of mouse pregnancy. Pumps were inserted into a subcutaneous pocket created in the hip space. The pumps contained either the $\mathrm{TXA}_{2}$-analog U-46619 (Cayman Chemical) dissolved in $0.5 \%$ ethanol or $0.5 \%$ ethanol (vehicle) which was continuously infused at $2000 \mathrm{ng} / \mathrm{h}$ throughout the remainder of pregnancy (Fung et al., 2011). Previous model characterization has shown that plasma 11-dehydrothromboxane $B_{2}$ levels were similar between the vehicle and U-46619 exposed fetuses, providing evidence that U-46619 did not cross the placenta to affect the pups directly (Fung et al., 2011). Following spontaneous delivery, pups were weighed on postnatal day (P)0. Pups born to dams receiving $\mathrm{TXA}_{2}-$ analog and weighing $<1.266 \mathrm{~g},<10$ th percentile for weight based on sham pup weights, were assigned to the IUGR group. Using this cutoff, approximately one-third of $\mathrm{TXA}_{2}-$ analog pups were defined as small for gestational age (SGA), which is similar to the incidence of human SGA infants, born to mothers with uteroplacental insufficiency in IUGR epidemiological studies (Eskenazi et al., 1993). Pups born to dams receiving $0.5 \%$ ethanol and weighing $>1.266$ $g$ (>10th percentile) were assigned to the vehicle group. All pups were cross-fostered to unmanipulated mouse dams postdelivery to minimize the surgical effects of pump insertion in the birth dams.

\section{Postnatal hyperoxia exposure}

Litters of vehicle and IUGR pups were placed in either $75 \%$ oxygen (hyperoxia) in a Plexiglas chamber (Biospherix) or $21 \%$ oxygen (room air) within $24 \mathrm{~h}$ after birth for $14 \mathrm{~d}$ (Aslam et al., 2009; Lee et al., 2014). Exposure to hyperoxia was continuous, with brief interruption only for animal care $(<10 \mathrm{~min} / \mathrm{d})$. The concentration of oxygen was maintained with an oxygen controller (ProOx, Biospherix). Ventilation within the chamber was adjusted to remove $\mathrm{CO}_{2}$ such that it did not exceed $0.5 \%$. A hygro-thermometer was used in the chamber to monitor temperature and humidity. Temperature in the chamber did not exceed $23^{\circ} \mathrm{C}$ and humidity level was maintained using dishes of desiccant in the bottom of the chamber. A foster dam was placed in the hyperoxia chamber with each vehicle or IUGR litter, and foster dams were rotated from hyperoxia to room air every $24-48 \mathrm{~h}$ to prevent excessive oxygen toxicity to the adult animals. Litters were removed from the hyperoxia chamber at $14 \mathrm{~d}$ and euthanized for tissue collection.

\section{RNA sequencing, analysis, and bioinformatics}

P14 pups were decapitated, whole brains were removed, and right hemispheres were immediately placed in RNAlater solution (Invitrogen) and stored at $-80^{\circ} \mathrm{C}$ until RNA extraction. Total RNA was isolated using the mirVana MiRNA Isolation kit (Thermo Fisher Scientific) per manufacturer protocol and quantified with NanoDropTM Spectrophotometer (Thermo Fisher Scientific). Samples were submitted to Northwestern University Feinberg School of Medicine NUSeq Core facility for TruSeq stranded mRNA sequencing library preparation and HiSeq high throughput sequencing using the Illumina platform (Illumina). For pooled samples, two samples were submitted for each of the four experimental groups, making a total of eight samples submitted for RNA sequencing. Pooled RNA samples consisted of a mix of sex with two to three animals per sample. For individual, non-pooled, RNA-seq, sample sizes were as follows: vehicle/normoxia (control) $n=7$ (four females, three males), IUGR/normoxia $n=5$ (one female, four males), vehicle/hyperoxia $n=7$ (two females, five males), IUGR/hyperoxia $n=8$ (three females, five males). To assess purity, each RNA sample underwent Bioanalyzer analysis; an RNA Integrity Number score of 7 or higher was used to indicate sufficient quality to proceed with library construction. The RNA samples were enriched for mature RNA and fragmented to obtain RNA fragments $\sim 50 \mathrm{bp}$ in size. A cDNA library was prepared with adapters added for paired end sequencing on the Illumina platform.

Sequence analysis and bioinformatics was conducted by the Advanced Bioinformatics and Bio-Computation Core Facility at the Center for Genetic Medicine at Northwestern University. The quality of DNA reads, in fastq format, was evaluated using FastQC (Babraham Bioinformatics, Babraham Institute, Cambridge, UK). Adapters were trimmed and reads of poor quality or aligning to rRNA sequences were filtered. The cleaned reads were aligned to the Mus musculus genome (mm10) using STAR (Dobin et al., 2013) and Ceto (https://github. com/ebartom/NGSbartom). Read counts for each gene were calculated using HTSeq-Counts (Anders et al., 2014) in conjunction with a gene annotation file for mm10 obtained from UCSC (University of California Santa Cruz; http://genome.ucsc.edu). A comprehensive QC report was generated using MultiQC (Ewels et al., 2016). Differential expression was determined using 
DESeq2 (Love et al., 2014). The cutoff for determining significantly differentially expressed genes (DEGs) was a false discovery rate (FDR)-adjusted $p<0.05$.

For subanalysis, gene expression results were also categorized by brain cell type using a transcriptome database created by the Barres lab at Stanford University (Zhang et al., 2014). RNA sequencing of purified neurons, astrocytes, microglia, endothelial cells, pericytes, and various maturation states of oligodendrocytes from mouse cortex were used to generate this high-resolution transcriptome database of $>22,000$ genes.

Co-expression analysis was performed using the CoExpression Molecules identification Tool (CEMiTool) package in Bioconductor using variance stabilizing transformation and an FDR cutoff of 0.05 (Russo et al., 2018). IPA (QIAGEN) was used to identify significant biological pathways from the RNA-seq datasets (Krämer et al., 2014). A list of detected genes and detected proteins was used as the data input, using a $q<0.05$ cutoff for the gene pathway and $p<0.1$ cutoff for the protein pathway analyses, such that only significant genes/proteins were considered for significant pathways. The "user dataset" option was chosen to use each individual detected gene/ protein data set as the "reference set" for which to generate significant pathways. Pathways from the "diseases and biological functions" category were used for comparison analyses. Fisher's $t$ test of $p<0.05$ was used to determine statistical significance of a pathway.

\section{Validation of gene expression}

To validate RNA-seq results, qRT-PCR was performed on five genes related to myelination that were found by RNA-seq to be significantly changed. Total RNA from the right hemisphere at P14 was isolated and quantified as described above: vehicle/normoxia (control): $n=15$ (seven females, eight males), IUGR/normoxia: $n=10$ (four female, six males), vehicle/hyperoxia: $n=12$ (five females, seven males), IUGR/hyperoxia: $n=14$ (seven females, seven males). cDNA was prepared using the TaqMan Advanced miRNA cDNA Synthesis kit (Applied Biosystems). The following Taqman Advanced 20× Assays were used: Mobp (mm02745649_m1), Mbp (mm01266402_m1), Cnp (mm01306640_m1), Mog (mm00447824_m1), and Plp1 (mm01297210_m1), and Gapdh (mm99999915_g1; Applied Biosystems) as loading control. Real-time PCR was performed using Bio-Rad CFX Real Time PCR Detection System and Software (Bio-Rad Laboratories). Technical replicates of four were used for each sample. Relative gene target amounts were normalized to the housekeeping gene Gapdh using the $\Delta \Delta C T$ method (Livak and Schmittgen, 2001). Regression was used to compare estimates of fold difference between RNA-seq and validation using Prism, version 7.0 (GraphPad Software Inc).

\section{Region of interest analysis}

Tissue from the following four brain regions were collected for analysis. Corpus callosum: control: $n=7$ (two female, five male), IUGR/normoxia: $n=9$ (four female, five male), vehicle/hyperoxia: $n=8$ (four female, four male),
IUGR/hyperoxia: $n=6$ (four female, one male); internal capsule: control: $n=6$ (two female, four male), IUGR/normoxia: $n=8$ (three female, five male), vehicle/hyperoxia: $n=8$ (four female, four male), IUGR/hyperoxia: $n=6$ (three female, three male); subcortical white matter (SCWM): control: $n=5$ (two female, three male), IUGR/normoxia: $n=4$ (two female, two male), vehicle/hyperoxia: $n=4$ (1 female, three male), IUGR/hyperoxia: $n=6$ (five female, one male); and cerebellum: control: $n=4$ (four male), IUGR/normoxia: $n=7$ (two female, five male), vehicle/hyperoxia: $n=7$ (three females, four males), IUGR/hyperoxia group: $n=6$ (three female, three male). Mice were deeply anesthetized, then decapitated, brains were removed and chilled in ice-cold RNAlater solution (Invitrogen) for $5 \mathrm{~min}$, cut into 1-mm sections using a stainless-steel mouse brain slicer, then white matter regions of interest were dissected using tungsten needles. Dissected region of interest tissue was placed in RNAlater and stored at $-80^{\circ} \mathrm{C}$ until RNA extraction. Total RNA isolation, cDNA synthesis, and quantitative RT-PCR were performed as described above. Taqman Advanced 20× Assays for Mobp, Mbp, Cnp, Mog, Plp1, and Gapdh were used as for entire hemisphere above.

\section{Statistical analysis}

Four to fifteen mice were used per treatment group. Mice from both sexes were used. In order to account for the intrauterine environment as a cofounder in developmental studies, mice were taken from at least two independent litters. The comparative $\mathrm{C}_{\mathrm{T}}$ method $\left(\Delta \Delta \mathrm{C}_{\mathrm{T}}\right.$ method) was used to analyze relative gene expression changes from qRT-PCR data (Livak and Schmittgen, 2001). Statistical significance and SEM for $q R T-P C R$ data were calculated from $\Delta \mathrm{C}_{\mathrm{T}}$ values. To compare two groups (experimental to control) Mann-Whitney test was used, given assumption of non-Gaussian distribution. Statistical analyses were conducted using GraphPad Prism version 7.0 (GraphPad Software). All data are expressed with n representing the number of animals and with significance at $p<0.05$.

\section{Results}

\section{IUGR, hyperoxia, and IUGR/hyperoxia have distinct effects on the brain transcriptome}

To test whether IUGR and postnatal hyperoxia alters expression of genes associated with oligodendrogliogenesis or myelination, we performed RNA-seq in a pilot experiment using total RNA pooled from a small number of P14 brains. P14 was chosen as it is the midpoint of myelination in rodents and allowed for sufficient postnatal hyperoxia exposure. We performed differential expression analysis (DEA) and compared three experimental groups against control: IUGR, hyperoxia, and IUGR/hyperoxia (Fig. 1A,B). We observed distinct gene expression between groups (Fig. $1 A-C$ ). IUGR had the fewest DEGs compared with control (69; FDR adj $p<0.05$ ), while hyperoxia had the greatest (1924). IUGR/hyperoxia had 647 DEGs (Fig. 1C,D). There was minimal overlap between groups, and only 18 DEGs common to all. Top 20 
A

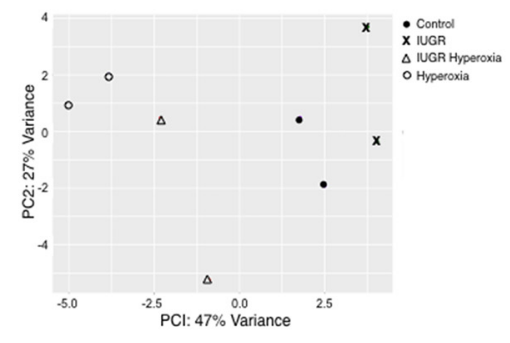

D

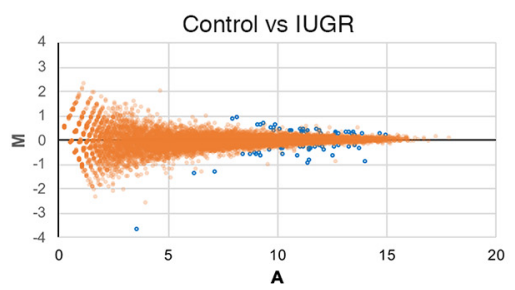

B

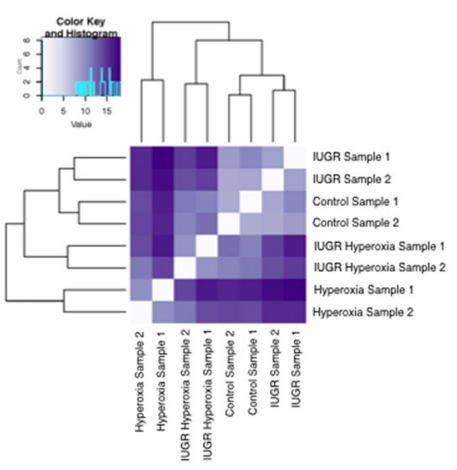

C

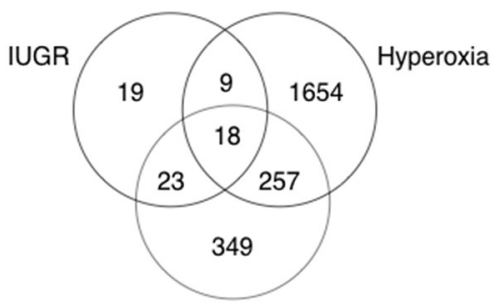

IUGR with Hyperoxia
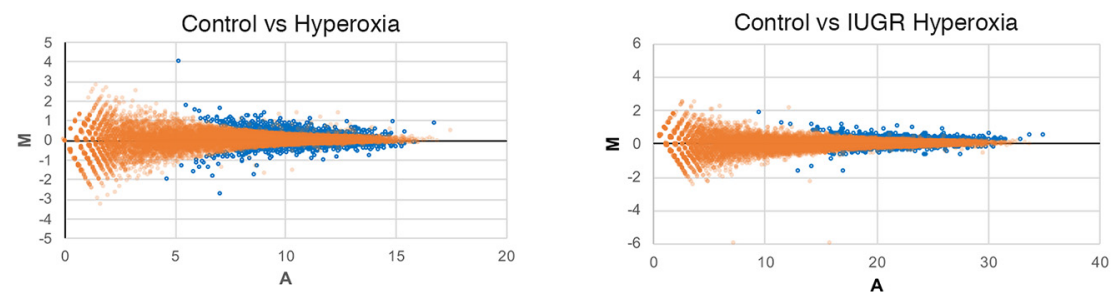

Figure 1. Comparison of RNA-seq gene expression data between control, IUGR, hyperoxia, and IUGR/hyperoxia. $\boldsymbol{A}$, PCA plot of gene expression showing variance between groups, $n=2$ pooled samples. $\boldsymbol{B}$, Heatmap demonstrating difference in gene expression between groups/samples. Dark purple represents most variance and white least variance. $\boldsymbol{C}$, Venn diagram of significant DEGs in IUGR, hyperoxia, and IUGR/hyperoxia (FDR adj $p<0.05$ ). $\boldsymbol{D}$, MA plots showing relationship between control versus IUGR, control versus hyperoxia, and control versus IUGR/hyperoxia. M (log ratio) and A (mean of normalized read counts). Orange dots represent genes that are not significantly different and blue spots represent genes that are significantly different $(p<0.05)$.

upregulated and downregulated DEGs also differed between groups, including directionality of expression, with prominent downregulation in IUGR/hyperoxia (Fig. 2).

\section{IUGR and hyperoxia have different effects on OL genes}

To determine whether expression changes lead to WMI in the three groups, we evaluated DEGs specifically expressed by oligodendroglia (OLDEGs) in the pooled data set. DEGs were categorized by brain cell type using a transcriptome database (Zhang et al., 2014). We found that IUGR, hyperoxia, and IUGR/hyperoxia demonstrated distinct patterns (Fig. 3A). IUGR yielded 6 OLDEGs, hyperoxia yielded 113, while IUGR/hyperoxia yielded 80 , with minimal overlap between groups. Notably, 63 OLDEGs were exclusively altered in hyperoxia and $28 \mathrm{ex}-$ clusively expressed in IUGR/hyperoxia. We next categorized OLDEGs by when in the lineage expression occurred, e.g., mature myelinating OLs, myelinating/ newly formed OLs, newly formed OLs, OPCs, or expression seen at all stages of differentiation (Table 1). IUGR showed upregulation of 6 OLDEGs throughout the lineage (Fig. 3B). Hyperoxia demonstrated predominant downregulation of OLDEGs in myelinating/newly formed OLs (Fig. 3C). IUGR/hyperoxia demonstrated downregulated OLDEGs primarily in later maturation stages (Fig. $3 D$ ), $75 \%$ of genes expressed exclusively by mature myelinating and myelinating/newly formed OLs. DEGs with the largest magnitude Log2 fold change and lowest FDR adjusted $p$ values were myelin specific: $M o B P$, Plp1, Mog, and Cnp (Table 2).

\section{Hyperoxia with and without IUGR decreases myelin gene network expression}

We repeated the RNA-seq using a larger sample size of individual (non-pooled) samples. Focusing on the IUGR/ hyperoxia data, as our pooled samples had shown the greatest effect on WMI in this group, we again found distinct gene expression compared with control (Fig. 4A). While DEA identifies a large number of genes that differ between groups, it does not give information on interconnections between DEGs. To address this, we performed unsupervised gene co-expression analysis on the nonpooled RNA sequencing data with CEMiTool (Russo et al., 2018). CemiTool generated five modules highly correlated with the data set (Fig. 4B). Module 4 (M4) was significantly enriched with 134 genes that were identified to be related to myelination by the hub genes: MoBP, Plp1, Gsn, and Mog (Fig. 4B). Notably, activity in M4 was lower in both hyperoxia and IUGR/hyperoxia (Fig. $4 C, D$ ) as demonstrated by statistically significant adjusted $p$ values and normalized enrichment scores (Table 3). These findings support the results from the DEA performed on our pooled samples and add further evidence that specific myelin genes are differentially expressed following these exposures (Chang et al., 2018). 

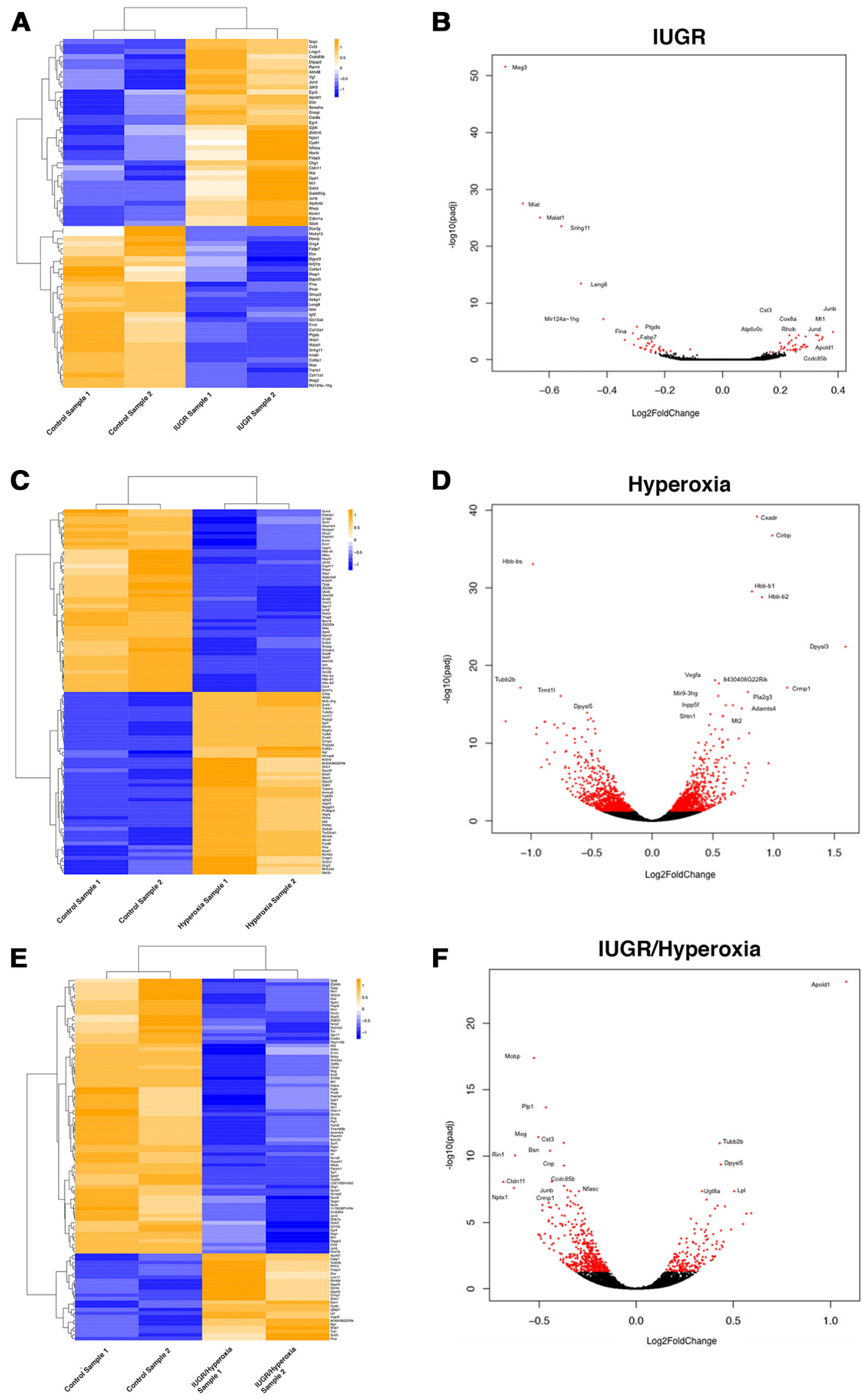

Figure 2. Comparison of significant DEGs in IUGR, hyperoxia, and IUGR/hyperoxia to control (FDR adj $p<0.05)$. $\boldsymbol{A}$, Heatmap of all significant DEGs between control and IUGR samples. Genes with similar expression patterns are clustered together (upregulated genes are dark orange and downregulated genes are dark blue). B, Volcano plot of all DEGs in IUGR (significant DEGs with top 20 DEGs are red and labeled, non-significant DEGs in dataset are black). $\boldsymbol{C}$, Heatmap of top 100 significant DEGs between control and hyperoxia samples. $\boldsymbol{D}$, Volcano plot of all DEGs in hyperoxia group [top 25 significant (red) DEGs are red and labeled]. $\boldsymbol{E}$, Heatmap 
continued

of top 100 significant DEGs between control and IUGR/hyperoxia samples. $\boldsymbol{F}$, Volcano plot of all DEGs in IUGR/hyperoxia group (top 25 significant DEGs are red and labeled).

\section{Unique gene regulators identified in hyperoxia and IUGR/hyperoxia}

We applied IPA to our non-pooled dataset to determine potential gene relationships and upstream regulators (Krämer et al., 2014). Using $p<0.05$ and the additional cutoff of predicted $z$ score $\geq 2$ or $\leq-2$, IPA identified no upstream regulators in IUGR, 5 upstream regulators in hyperoxia (Fig. 5A), and 25 in IUGR/hyperoxia (Fig. 5B). TCF7L2 was the only regulator in common between hyperoxia $(p=1.95 \mathrm{E}-12, z=-4.375)$ and IUGR/hyperoxia $(p=3.97 \mathrm{E}-8, z=-4.28)$. The Regulator Effects algorithm in IPA was next applied to the IUGR/hyperoxia data. This algorithm connects upstream regulators, dataset molecules and downstream functions/diseases, to generate hypotheses that can explain how the activation/inhibition of an upstream regulator affects downstream target molecule expression, and the impact of molecular expression on functions/diseases (Krämer et al., 2014). In addition to
TCF7L2, predicted upstream regulators BDNF $(p=0.0002, z=-2.189)$, SOX2 $(p=5.64 \mathrm{E}-5, z=-1.672)$, MYOC $(p=0.0006, z=-1)$, DGCR8 $(p=5.93 \mathrm{E}-8, z=$ $-1.369)$, and FMR1 $(p=2.9 \mathrm{E}-10, z=3.578)$ were suggested to inhibit myelination (Fig. $5 \mathrm{C}$ ).

\section{Perturbed myelin gene expression varies regionally in IUGR, hyperoxia, and IUGR/hyperoxia}

To validate RNA-seq results, qRT-PCR was performed on four myelin genes that were found to be significantly downregulated in DEA: MoBP, Plp1, Mog, and Cnp. Using total RNA from control and IUGR/hyperoxia P14 hemispheres, we found all genes to be significantly downregulated, ${ }^{*} p<0.05$ (Fig. 6). To determine whether specific white matter regions had greater myelin gene downregulation, we collected total RNA from four regions of interest at P14 for qRT-PCR: corpus callosum,
A

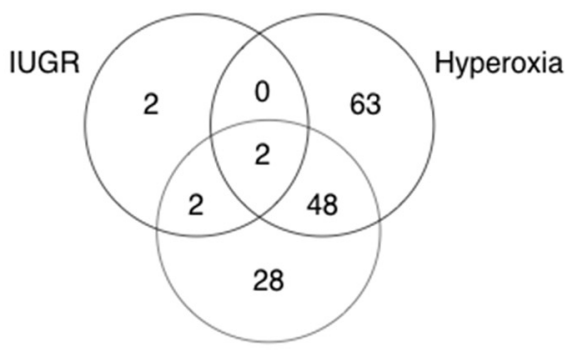

IUGR with Hyperoxia
B

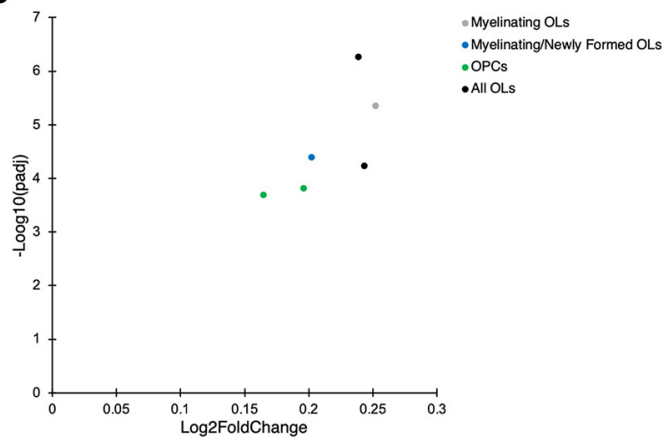

C

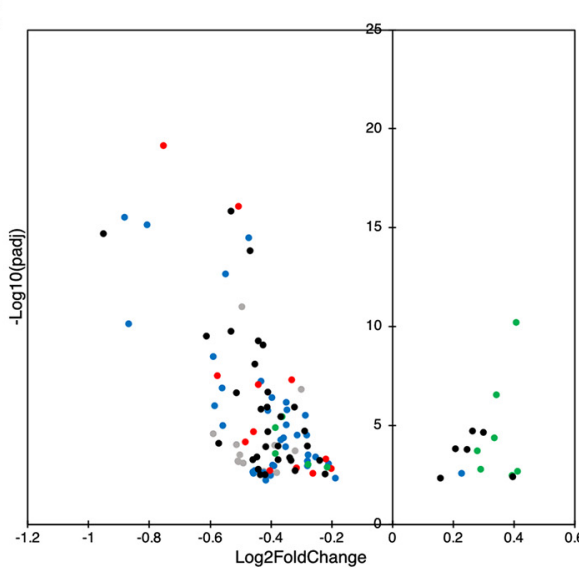

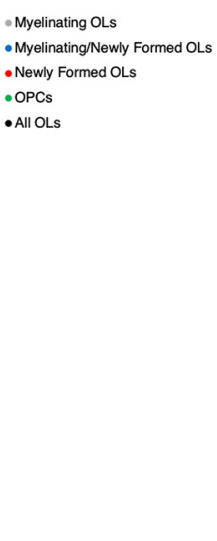

D

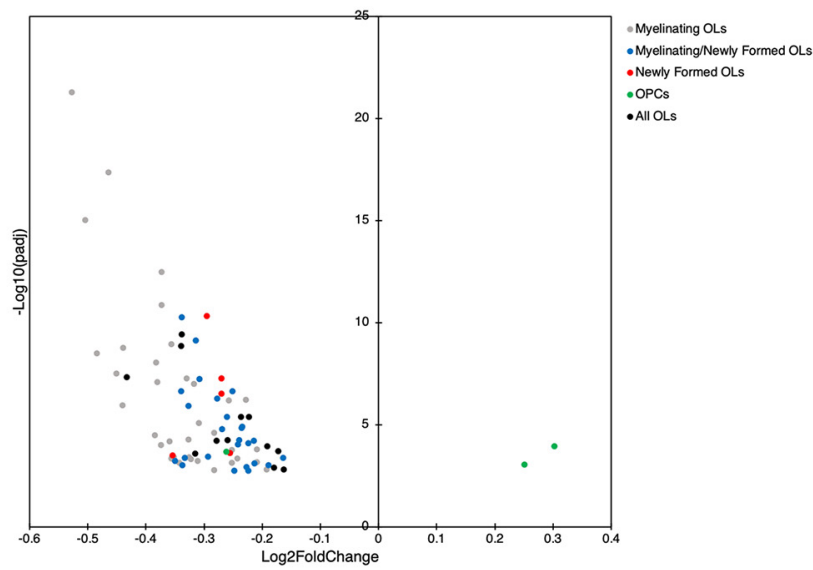

Figure 3. Comparison of differentially expressed OL genes (OLDEGs) in IUGR, hyperoxia, and IUGR/hyperoxia. $\boldsymbol{A}$, Venn diagram of significant OLDEGs in IUGR, hyperoxia, and IUGR/hyperoxia (FDR adj $p<0.05$ ). $\boldsymbol{B}$, Scatter plot of significant OLDEGs in IUGR. Fold difference between log2 normalized expression in IUGR and control plotted versus -log10 adjusted $p$ value. OLDEGs subcategorized by type of cell in OL lineage they are expressed by (gray: myelinating OL, blue: myelinating/newly formed OLs, red: newly formed OLs, green: OPCs, or black: all OLs). C, Scatter plot of significant OLDEGs in hyperoxia. $\boldsymbol{D}$, Scatter plot of significant OLDEGs in IUGR/hyperoxia. 
Table 1: DEGs categorized by OL cell type in IUGR, hyperoxia, and IUGR/hyperoxia compared with control

\begin{tabular}{lll}
\hline Experimental group & Type of OL cell & Number of genes (percentage) \\
\hline IUGR & Mature myelinating OL & $1 / 6(16.67 \%)$ \\
& Myelinating/Newly formed OL & $1 / 6(16.67 \%)$ \\
& Newly formed OL & $0 \%)$ \\
& OPCs & $2 / 6(33.33 \%)$ \\
& All OL (non-specific expression) & $2 / 6(33.33 \%)$ \\
Hyperoxia & Mature myelinating OL & $15 / 113(13.27 \%)$ \\
& Myelinating/Newly formed OL & $37 / 113(32.74 \%)$ \\
& Newly formed OL & $10 / 113(8.85 \%)$ \\
IUGR with hyperoxia & OPCs & $14 / 113(12.39 \%)$ \\
& All OL (non-specific expression) & $37 / 113(32.74 \%)$ \\
& Mature myelinating OL & $35 / 80(43.75 \%)$ \\
& Myelinating/Newly formed OL & $25 / 80(31.25 \%)$ \\
& Newly formed OL & $5 / 80(6.25 \%)$ \\
& OPCs & $3 / 80(3.75 \%)$ \\
\end{tabular}

internal capsule, SCWM, and cerebellum, then assessed expression of MoBP, Plp1, Mog, Cnp, and Mbp. Variable expression was seen in different regions for each treatment group. IUGR and hyperoxia showed statistically significant downregulation of OL genes in the corpus callosum and internal capsule that were not seen in entire hemisphere analysis (Fig. 7A,B). Surprisingly, IUGR/hyperoxia did not demonstrate significantly changed expression in white matter regions, although there was significant downregulation of MoBP in SCWM and Cnp in the cerebellum (Fig. 7C).

\section{Difference in myelin gene expression between sexes}

To evaluate the impact sex has on WMI in IUGR and hyperoxia, qRT-PCR data for MoBP, Plp1, Mog, and Cnp from P14 hemispheres was separately analyzed by sex. There were no significant differences found between sexes in the control group. Nor were there significant differences between sexes found in IUGR, but there was a trend toward decreased myelin gene expression in females compared with males for all four genes (Fig. 8A). Hyperoxia showed decreased myelin gene expression in females compared with males that was statistically significant for Plp1 $(p=0.01)$; the other three genes showed a trend toward decreased expression in females compared with males (Fig. 8B). In contrast, IUGR/hyperoxia showed

Table 2: Top 10 differently expressed myelin genes in IUGR/ hyperoxia compared with control in RNASeq dataset

\begin{tabular}{lll}
\hline Gene symbol & Log2 fold change & FDR adj $p$ value \\
\hline Mobp & -0.526952566 & $5.26 \mathrm{E}-22$ \\
Plp1 & -0.464800028 & $4.34 \mathrm{E}-18$ \\
Mog & -0.50406988 & $9.40 \mathrm{E}-16$ \\
Cnp & -0.372706581 & $3.38 \mathrm{E}-13$ \\
Mag & -0.355944387 & $1.13 \mathrm{E}-09$ \\
Myrf & -0.329926462 & $5.55 \mathrm{E}-08$ \\
Mal & -0.384694903 & $3.41 \mathrm{E}-05$ \\
Mbp & -0.326101762 & 0.000401 \\
Opalin & -0.311350925 & 0.000604 \\
Omg & -0.19212208 & 0.00159 \\
\hline
\end{tabular}

statistically significant decreases in Plp1 $(p=0.0006)$ and $C n p(p=0.0076)$ in males compared with females (Fig. 8C).

\section{Discussion}

WMI places IUGR infants at a higher risk of developing severe motor dysfunction including CP. Previous preclinical studies have shown that IUGR and postnatal oxygen exposure, individually and in combination, have adverse effects on the developing brain and result in WMI (Gerstner et al., 2008; Schmitz et al., 2011; Tolcos et al., 2011, 2017; Reid et al., 2012; Ramani et al., 2013; Ritter et al., 2013; Chang et al., 2018). Using a mouse model of placental insufficiency (Fung et al., 2011), we found distinct transcriptomic changes in the brain in three experimental groups: IUGR, hyperoxia, and IUGR/hyperoxia. Here, we observed that each group resulted in differences in gene transcription relating to white matter development and myelination. IUGR, hyperoxia, and IUGR/hyperoxia differed in the number and type of affected genes with minimal overlap in DEGs between each group. As all three study groups have previously shown altered myelination to some degree (Chang et al., 2018), it was somewhat surprising to find minimal overlap in differential gene expression. Additionally, the directionality of DEGs differed between groups, with prominent downregulation of genes in IUGR/hyperoxia that was not seen in IUGR nor hyperoxia alone. This finding suggests that disruption of white matter development likely occurs through distinct mechanisms and cellular interactions in each perturbation to the brain.

Distinct transcriptomic changes between groups were also seen in subanalysis of oligodendrocyte specific gene expression. Similar to what was seen when all cell types were examined, there was minimal overlap in OLDEGs between IUGR, hyperoxia, and IUGR/hyperoxia. The pattern of expression again showed predominant downregulation of OLDEGs in IUGR/hyperoxia. The predicted type of OL cell that was most affected also differed between groups. In hyperoxia, DEGs specific to newly formed/myelinating OLs were found to be the most affected. This is consistent with other studies showing transient loss of these cell 
A

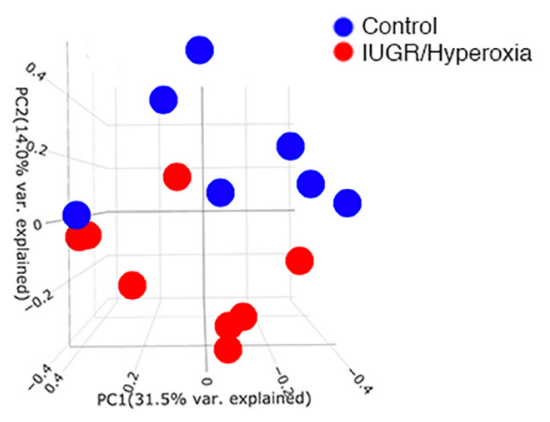

B

Phenotypes: 1 Hyperoxia $\square$ Control $\square$ IUGR/Hyperoxia $\square$ IUGR
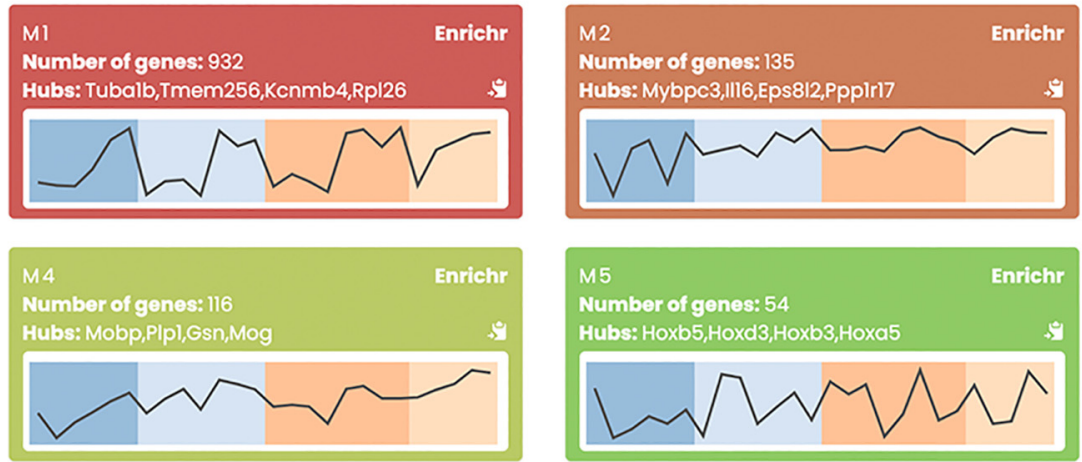

IUGR/Hyperoxia

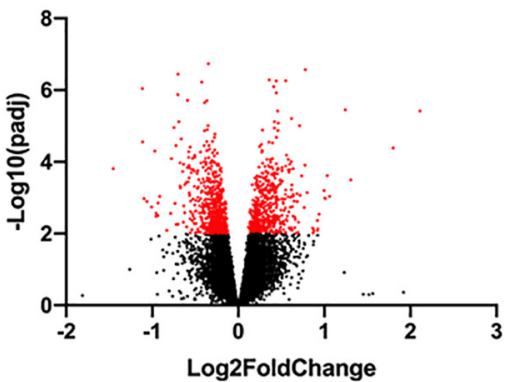

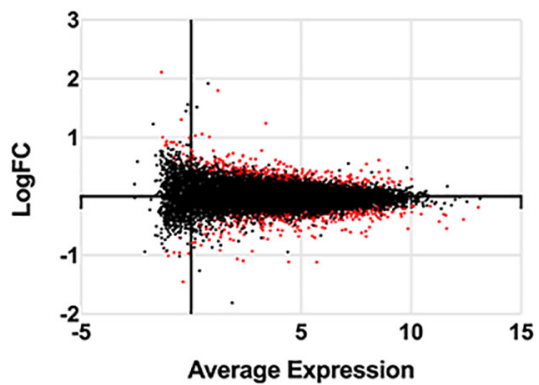

C

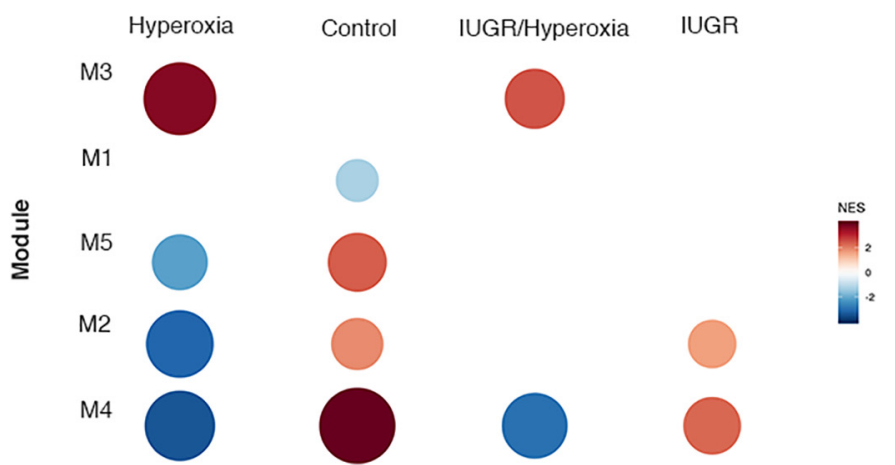

D

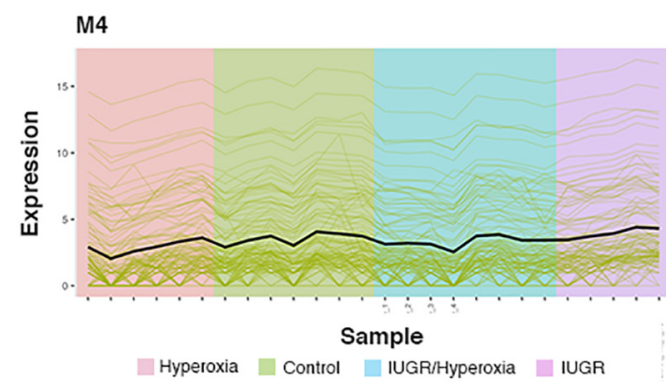

Hyperoxia Control IUGR/Hyperoxia IIUGR

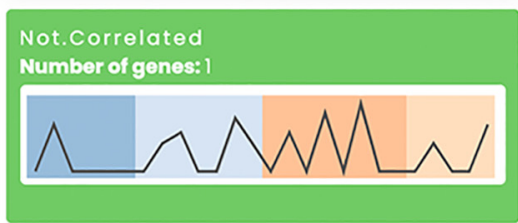

Figure 4. Differential gene analysis and co-expression analysis of expanded non-pooled data. $\boldsymbol{A}$, PCA plot comparing control versus IUGR/hyperoxia samples (control $n=7$; IUGR/hyperoxia $n=8$ ). Volcano plot of all DEGs in IUGR/hyperoxia group, significant DEGS $(p<0.05)$ are in red. MA plot showing relationship between control versus IUGR/hyperoxia. red represents genes that are significantly different $(\boldsymbol{p}<0.05)$. B , CemiTool Module profile plots for modules M1-M6. Number of genes and hub genes displayed for each module. Black line in each plot indicates median expression activity of genes in the module. Experimental groups are color coded. C, Gene set enrichment analysis showing the M4 module activity on each class of samples. The size and color of the circle represents the normalized enrichment score (NES). $\boldsymbol{D}$, Profile plot for M4. The black line represents the median expression activity of all genes in the module. Samples are shown in the $x$-axis and colors represent the different experimental groups.

Table 3: Co-expression modules (M1-M5) with adjusted $p$ values and normalized enrichment scores (NES) for control, IUGR, hyperoxia, and IUGR/yyperoxia

\begin{tabular}{llllllrrr}
\hline Module & $\begin{array}{lllll}\text { Control } \\
\text { adj } p \text { value }\end{array}$ & $\begin{array}{l}\text { Control } \\
\text { NES }\end{array}$ & $\begin{array}{l}\text { Hyperoxia } \\
\text { adj } p \text { value }\end{array}$ & $\begin{array}{l}\text { Hyperoxia } \\
\text { NES }\end{array}$ & $\begin{array}{l}\text { IUGR/hyperoxia } \\
\text { adj } p \text { value }\end{array}$ & $\begin{array}{l}\text { IUGR/hyperoxia } \\
\text { NES }\end{array}$ & $\begin{array}{l}\text { IUGR } \\
\text { adj } p \text { value }\end{array}$ & $\begin{array}{l}\text { IUGR } \\
\text { NES }\end{array}$ \\
\hline M1 & 0.00396 & -1.27 & 0.25 & 1.34 & 0.74373 & 0.94 & 1 \\
M2 & 0.00411 & 1.87 & 0.0002 & -3.12 & 0.0643 & 1.32 & 0.71 \\
M3 & 0.15473 & -1.21 & 0.00169 & 3.61 & 0.00053 & 2.47 & 0.00025 & 1.61 \\
M4 & 0.00396 & 3.94 & 0.0002 & -3.38 & 0.00413 & -2.95 & 0.625 & 0.00025 \\
M5 & 0.00396 & 2.38 & 0.0002 & -2.13 & 0.0643 & 1.4 & -6.83 \\
\end{tabular}


A

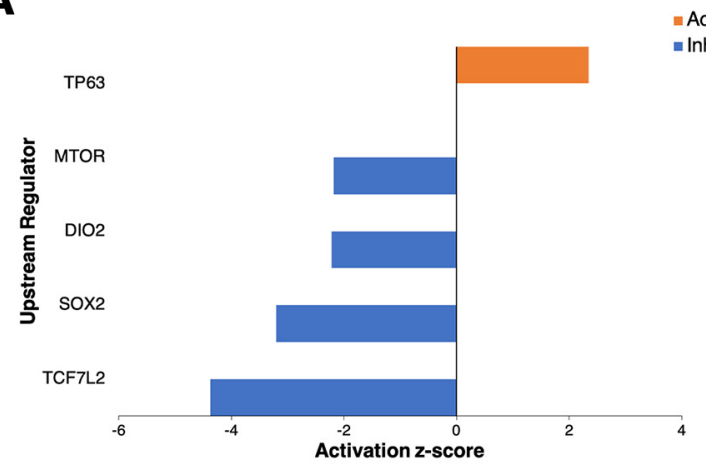

B

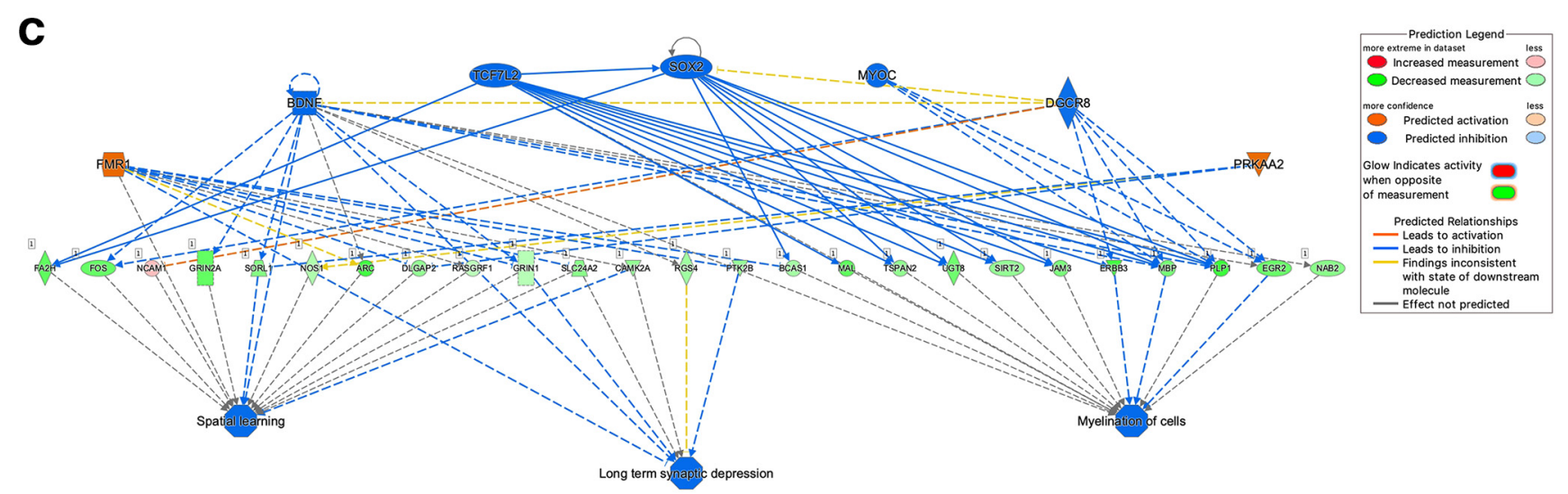

Figure 5. Upstream regulators identified with IPA from non-pooled RNA-seq data. $\boldsymbol{A}$, Upstream regulators identified in hyperoxia alone with $p$ value of overlap $<0.05$; orange: upstream regulators with $z$ scores $\geq 2$ (activated) and blue: upstream regulators with $z$ scores $\leq-2$ (inhibited). $\boldsymbol{B}$, Upstream regulators identified in IUGR/hyperoxia with $p$ value of overlap $<0.05$ and $z$ scores $\geq 2$ or $\leq-2$. $\boldsymbol{C}$, Regulator Effects identified altered regulators and networks in IUGR/hyperoxia. Upstream regulators are located at the top of the network, target genes are in the middle of network, and predicated disease or function in the bottom of network.

types both in culture and in rat pups (Gerstner et al., 2006, 2008; Brill et al., 2017). In IUGR/hyperoxia, statistically significant DEGs were characteristic of cells later in the OL lineage, suggesting a specific effect on myelinating OLs with the combination of exposures. In fact, the DEGs with the lowest FDR adjusted $p$ values and highest fold changes in the IUGR/hyperoxia dataset, before subanalysis, were myelin genes, including MoBP, Plp1, Mog, and Cnp. Unlike in IUGR/hyperoxia, the expression of these four myelin genes were not as significantly affected in IUGR or hyperoxia alone. This indicates that transcriptional changes in OLs are specific to the conditions of IUGR, hyperoxia, and IUGR/hyperoxia, lending insight into the differing type of WMI previously identified in these groups (Chang et al., 2018).

Of note, the majority of the significant DEGs in our DEA had fold changes $<2$ or $>-2$, a cutoff routinely used in
RNA sequencing studies. One explanation, is that the brain contains a multitude of different cell types and the effect on oligodendrocytes, primarily perturbed in WMI, would be diluted by these other cells $(\sim 80 \%)$ in the brain (ValerioGomes et al., 2018). As subtle changes in levels of RNAs can have biologically meaningful insights (Cantone et al., 2019), we chose to include all DEGs in our analysis if they satisfied the statistical cutoff of FDR adjusted $p<0.05$. In doing so, we were able to discover significant downregulation of DEGs specific to oligodendrocytes. This supported our hypothesis that gene expression changes occur in IUGR and hyperoxia to reflect the WMI previously reported (Chang et al., 2018). Given these promising results, we performed DEA and co-expression analysis, and applied IPA using RNA from an expanded nonpooled data set. The subsequent analyses provided us with information on gene networks and potential 
A

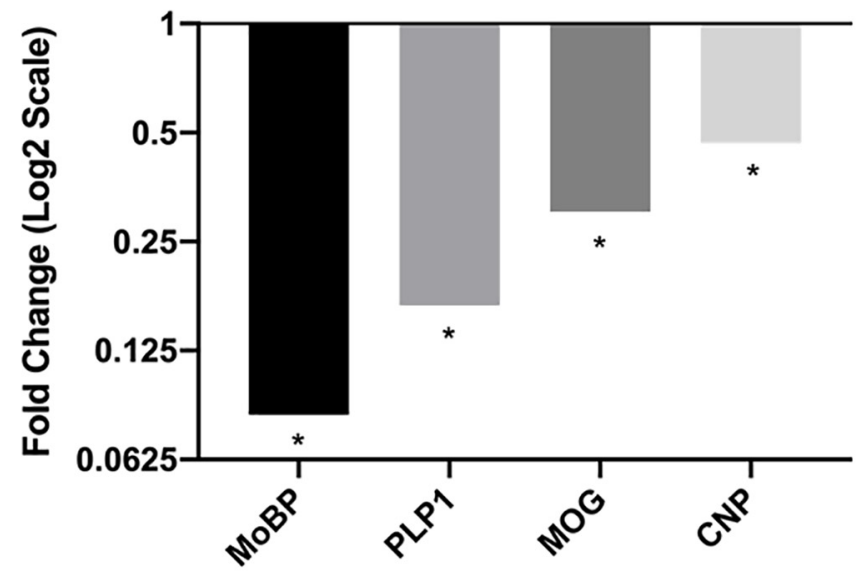

B

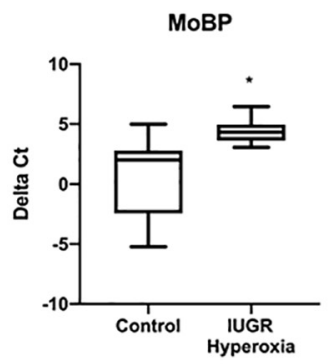

MOG

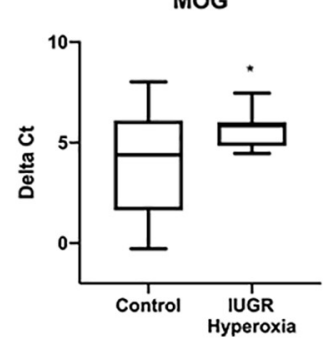

PLP1

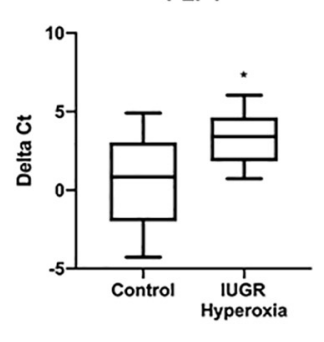

CNP

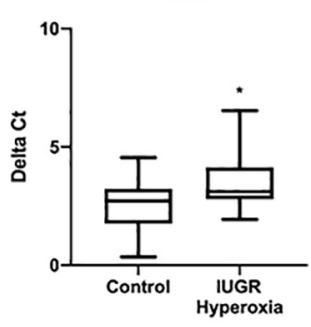

Figure 6. Validation of four most significantly downregulated myelin genes in IUGR/hyperoxia using qRT-PCR. $\boldsymbol{A}$, Relative fold change ( $y$-axis is log2 scale) of Mog, Plp1, MoBP, and Cnp in IUGR/hyperoxia versus control calculated using $\Delta \mathrm{C}_{\mathrm{T}} \mathrm{method}$ from qRT-PCR data (control $n=15$ and IUGR/hyperoxia $n=14$, significance ${ }^{*} p<0.05$ calculated from $\Delta \mathrm{C}_{\mathrm{T}}$ values). $\boldsymbol{B}$, Box plots showing $\delta \mathrm{Ct}\left(\Delta \mathrm{C}_{\mathrm{T}}\right)$ of $\mathrm{Mog}, \mathrm{Plp} 1, \mathrm{MoBP}$, and $\mathrm{Cnp}$ in control and IUGR/hyperoxia which were used to calculate statistical significance; Gapdh used as the normalization gene (control $n=15$ and IUGR/hyperoxia $n=14$, significance ${ }^{*} p<0.05$ ).

mechanisms, adding to the understanding of transcriptome changes occurring in IUGR and hyperoxia.

Unsupervised gene co-expression analysis provides information on the interconnections between the DEGs that cannot be determined by DEA alone. It creates networks of genes (modules) by using the fact that genes participating in the same molecular and biological processes tend to show highly correlated expression patterns (co-expression; van Dam et al., 2018). Co-expression analysis has provided important biological insights into infectious (Janova et al., 2016), inflammatory (Jochems et al., 2018), and neurologic disease (Voineagu et al., 2011). Co-expression analysis has also been shown to enhance gene relationships that are only seen as modest gene changes in DEA (Abbassi-Daloii et al., 2020). Analysis of our nonpooled RNA-Seq dataset identified a network of 134 genes enriched with genes primarily involved in myelination. This network was decreased in hyperoxia and IUGR/ hyperoxia compared with the control and IUGR groups. This supports our DEA results that genes related to myelination are specifically affected by hyperoxia and IUGR/ hyperoxia.

While co-expression networks are able to identify correlations, indicating which genes are active simultaneously and likely biologically related, they do not provide information about causality or distinguish between regulatory/ regulated genes (van Dam et al., 2018). Therefore, we next used IPA to identify potential upstream regulators and provide insight into the mechanism for WMI in the different exposure groups. In both hyperoxia and IUGR/hyperoxia, a pronounced downregulation of TCF7L2 signaling was seen. Downstream target molecules of TCF7L2 in our dataset included major myelin genes MoBP, Cnp, Mog, Plp1, and Mbp. TCF7L2 is a transcription factor specifically expressed in OLs during the time window that is critical for myelin formation, during the transition from OPCs to mature myelinating OLs (Fu et al., 2012; Lürbke et al., 2013; Hammond et al., 2015). It acts as a co-activator of $\beta$-catenin and is part of the canonical Wnt/ $\beta$-catenin pathway, a well-known signaling pathway involved in neurogenesis and OL maturation (Gaesser and FyffeMaricich, 2016). Inhibition of TCF7L2 in hyperoxia and IUGR/hyperoxia can therefore explain the specific downregulation we found in newly formed and myelinating OLs. Dysregulation of the Wnt pathway has been implicated in other types of perinatal WMI including $\mathrm{HI}$ encephalopathy, periventricular leukomalacia, and prematurity (Fancy et al., 2009; Back, 2017), and thus it is unsurprising that it may be involved in WMI secondary to hyperoxia and IUGR/hyperoxia.

Our study also supports, as has been previously demonstrated, that hyperoxia in isolation results in WMI (Gerstner et al., 2008; Ramani et al., 2013; Ritter et al., 2013; Chang et al., 2018). TCF7L2, SOX2 and mTOR, which are known to be important in normal myelination, made up three out of the four upstream regulators identified in the hyperoxia group as inhibited. The transcription factor SRY-box 2 (SOX2) has been shown to be involved in OL proliferation and differentiation during postnatal brain myelination (Hoffmann et al., 2014). SOX2 also plays a role in CNS remyelination after injury and acts by recruiting adult OPCs (Zhao et al., 2015). The mTOR/Akt pathway is a signaling pathway known to be integral in many aspects of OL development including OPC differentiation, myelination, and survival (Narayanan et al., 2009; Gaesser and Fyffe-Maricich, 2016). Several studies report that levels of both myelin mRNAs and proteins are reduced following inactivation of mTOR signaling (Bercury et al., 
A

IUGR: Corpus Callosum
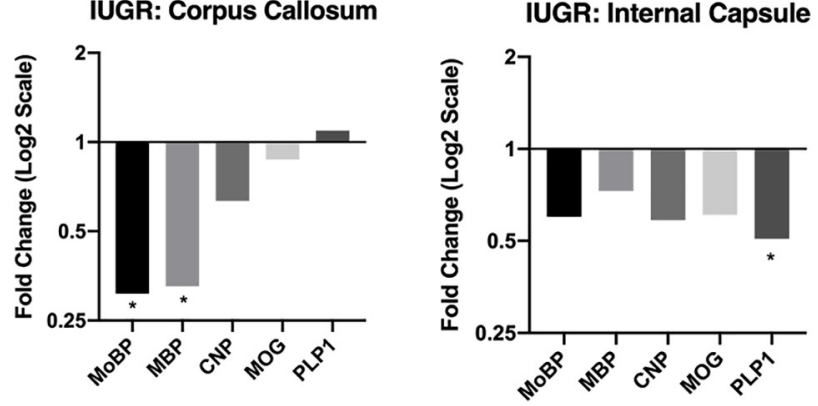

B

Hyperoxia: Corpus Callosum

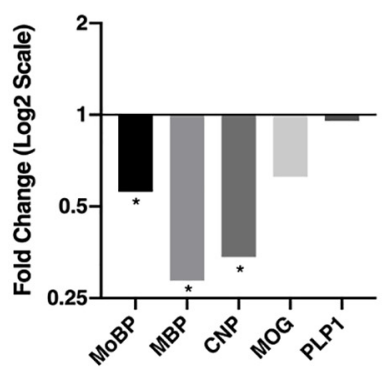

C

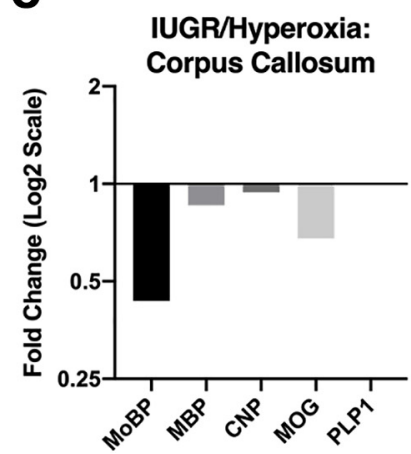

Hyperoxia: Internal Capsule
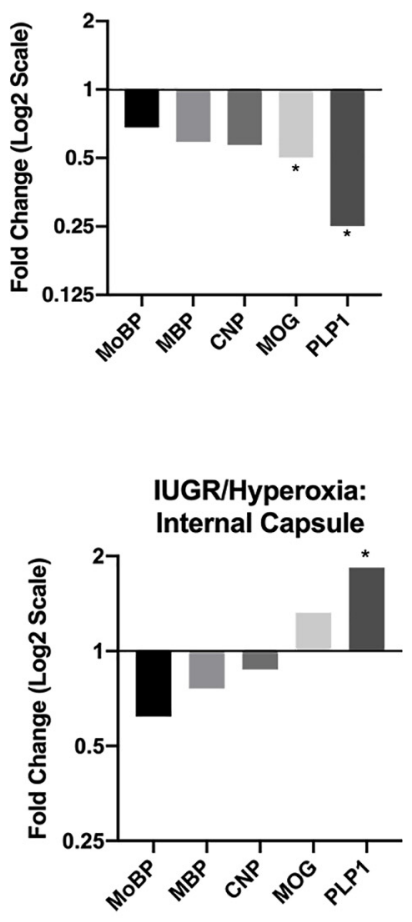

IUGR: SCWM

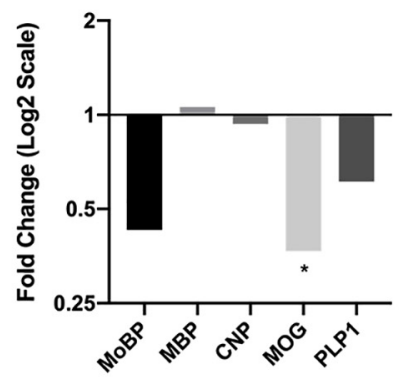

Hyperoxia: SCWM

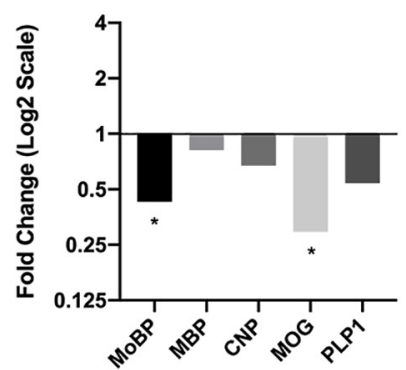

IUGR/Hyperoxia: SCWM

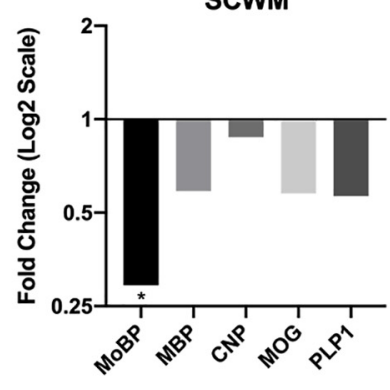

IUGR: Cerebellum

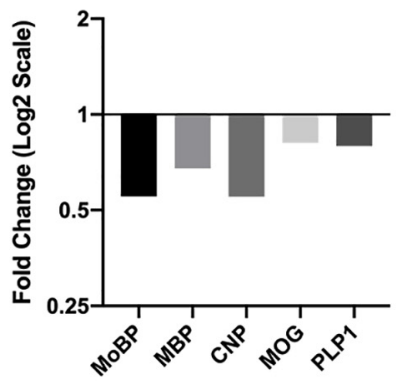

Hyperoxia: Cerebellum

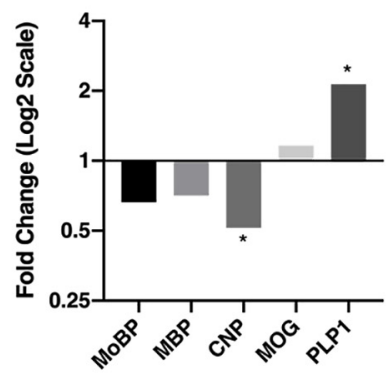

IUGR/Hyperoxia: Cerebellum

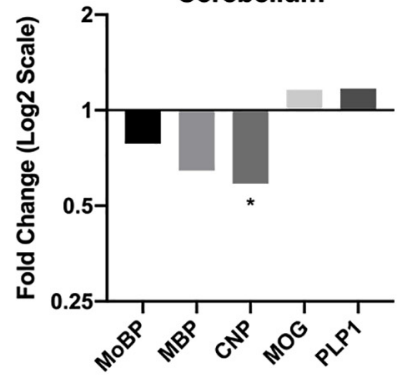

Figure 7. Myelin gene expression in different white matter regions of the brain (corpus callosum, internal capsule, SCWM, and cerebellum) measured with qRT-PCR at P14. A, Relative fold change (log2 scale) of MoBP, Mbp, Cnp, Mog, and Plp1 in IUGR versus control calculated using $\Delta \mathrm{C}_{\mathrm{T}}$ method. Corpus callosum (control $n=7$; IUGR $n=9$ ), internal capsule (control $n=6$, IUGR $n=8$ ), SCWM (control $n=5$, IUGR $n=4$ ), and cerebellum (control $n=4$, IUGR $n=7$ ), * $p<0.05$ calculated from $\Delta \mathrm{C}_{\mathrm{T}}$ values. $\boldsymbol{B}$, Relative fold change (log2 scale) of MoBP, Mbp, Cnp, Mog, and Plp1 in hyperoxia versus control. Corpus callosum (control $n=7$; hyperoxia $n=8$ ), internal capsule (control $n=6$, hyperoxia $n=8$ ), SCWM (control $n=5$, hyperoxia $n=7$ ), and cerebellum (control $n=4$, hyperoxia $n=7$ ), ${ }^{*} p<0.05$. C, Relative fold change (log2 scale) of MoBP, Mbp, Cnp, Mog, and Plp1 in IUGR/hyperoxia versus Corpus callosum (control $n=7$; IUGR/hyperoxia $n=6$ ), internal capsule (control $n=6$, IUGR/hyperoxia $n=6$ ), SCWM (control $n=5$, IUGR/hyperoxia $n=6$ ), and cerebellum (control $n=4$, IUGR/hyperoxia $n=6$ ), ${ }^{*} p<0.05$.

2014; Lebrun-Julien et al., 2014; Wahl et al., 2014). Our findings now add specific transcription factors and pathways that may be involved in WMI because of postnatal hyperoxia exposure.

In contrast to IUGR and hyperoxia alone, a much larger number of upstream regulators were identified in IUGR/ hyperoxia by IPA analysis. To understand the role of these additional upstream regulators in WMI, we used the regulator effects algorithm in IPA. This algorithm integrates results from the upstream regulator and downstream effects tools, to create hypotheses to explain how upstream regulators may cause a specific phenotype or outcome (Krämer et al., 2014). In addition to TCF7L2 and BDNF, which were identified in Upstream Regulator analysis, we were able to connect additional upstream effectors SOX2, MYOC, DGCR8, and FMR1, to the outcome of inhibited myelination. Similar to TCF7L2, the identification of BDNF involvement in IUGR/hyperoxia was unsurprising as this growth factor has been shown to pay a protective role in the neonatal brain following $\mathrm{HI}$ injury (Han and 
A

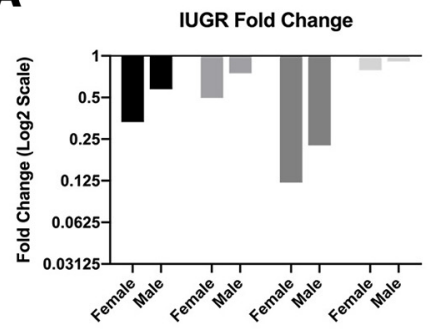

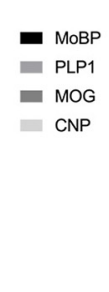

B

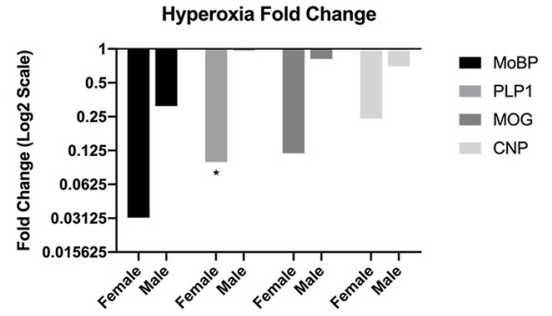

C

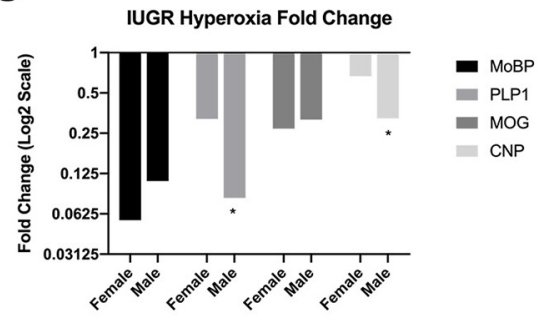

Figure 8. Sex comparison of myelin genes (MoBP, Plp1, Mog, Cnp) using qRT-PCR from RNA from P14 hemispheres. A, Relative fold change (log2 scale) of IUGR (female $n=8$, male $n=7$ ) versus control (females $n=7$, males $n=8$ ) calculated using $\Delta \mathrm{C}_{\mathrm{T}}$ method, female versus male comparison. $\boldsymbol{B}$, Relative fold change of hyperoxia (female $n=7$; male $n=5$; ${ }^{*} p<0.05$ ) versus control, female versus male comparison. $\boldsymbol{C}$, Relative fold change of IUGR/hyperoxia (female $n=7$, male $n=7$; ${ }^{*} p<0.05$ ) versus control calculated using $\Delta \mathrm{C}_{\mathrm{T}}$ method, female versus male comparison.

Holtzman, 2000; Chen et al., 2013). BDNF signals through oligodendrocyte-expressed TrkB that, in turn, activates the MAPK/Erk pathways to promote OL differentiation and myelination (Fletcher et al., 2018). Bdnf knock-out mice exhibit significant decreases in the expression of MBP and reduced mRNA transcripts of MBP and PLP in the hippocampus and cortex (Vondran et al., 2010; Gonsalvez et al., 2016; Fletcher et al., 2018). Additionally, BDNF heterozygous null mice demonstrate significant reductions in expression of MBP, PLP, MAG, and MOG in forebrain, corpus callosum, spinal cord and optic nerves (Djalali et al., 2005). The MAPK/Erk signaling is implicated as a late-stage regulator of CNS myelination (Gonsalvez et al., 2016; Ishii et al., 2019) and has also been shown to be important for myelin maintenance throughout adulthood (Ishii et al., 2012). Inhibition of BDNF in IUGR/hyperoxia therefore correlates with the specific downregulation of DEGs we found in myelinating/newly formed and mature myelinating OLs.

An additional interesting gene identified was Dgcr8 which regulates primary miRNA (miR) processing and has been found to be important in regulating progression of differentiation of oligodendroglia, myelin formation, and myelin maintenance (Lin et al., 2015). Alterations in miRs have been observed to change in several types of preterm brain injury. Differential expression of miRs was shown first in plasma of preterm infants with intraventricular hemorrhage (Chapman et al., 2018). Subsequently, changes in specific miRs within exosomes in CSF following posthemorrhagic hydrocephalus have been shown (Spaull et al., 2019). Proinflammatory miRs have also been found to correlate with oxidized hemoglobin metabolites and heme in CSF after intraventricular hemorrhage (Fejes et al., 2020). Additionally, miRs have been widely studied in $\mathrm{HI}$ encephalopathy in term neonates, in serum, dried blood spots and whole blood, as potential biomarkers of injury severity (Ponnusamy et al., 2016; Wang et al., 2018; Zhang et al., 2020). Specific to WMI, it was shown that mature miRs suppress the regenerative OL response to perinatal HI (Birch et al., 2014). Thus, differential expression of genes involved in miR processing is likely to have effects on white matter development.

These additional regulators identified in IUGR/hyperoxia by the regulator effector algorithm had $z$ scores that were not $\geq 2$ or $\leq-2$ so may otherwise have gone overlooked. Many of these upstream regulators converge to inhibit MBP and PLP1, which explains the pronounced downregulation of these genes in both DEA and co-expression analysis. The unique dysregulation identified by IPA at multiple points, from transcription, processing of $\mathrm{miR}$, and OL cellular signaling pathways in IUGR/hyperoxia, supports our idea of a multihit hypothesis of WMI with this combined insult. It also seems likely that each of these steps, that are known to be independently critical for myelination, are likely intertwined, and that appropriate and effective myelination occurs only when normal interactions are maintained.

We expected to see a more pronounced downregulation of myelin genes in IUGR/hyperoxia within isolated large white matter tracts, however, this was not observed. Instead, the most significant downregulated myelin genes, including MoBP, Plp1, Cnp, Mog, and Mbp, that we observed in entire hemisphere showed less significant changes in the corpus callosum, internal capsule, SCWM, and cerebellum. One potential explanation is that IUGR/hyperoxia affects the descending motor tracts, including the corticospinal tract, that run through the internal capsule later in development. A significant decrease in WM fiber length and volume in the internal capsule has been demonstrated on MRI with DTI at P28 in IUGR mice exposed to postnatal hyperoxia (Chang et al., 2018). Further investigation into the effect that timing of injury has on gene dysregulation would help us better understand the injury that occurs in this model. Specifically, postnatal hyperoxia exposure may affect gene expression differently, the longer the exposure time period. In addition, investigating different regions of the brain at earlier and later time points would also better define the injury that occurs in this model, as myelination of the developing brain occurs in the rostral to caudal direction. Thus, examination of a single time point is a limitation of this study.

Clinical and animal studies have shown that sex may play a role in neonatal neurodevelopmental outcomes. A number of clinical studies have identified male sex as an independent risk factor for poor neurodevelopmental outcomes in prematurity (Hintz et al., 2006; Sunny et al., 2020) and HI injury (Mirza et al., 2015; Narang et al., 2019). A sex bias has also been identified in children with 
$\mathrm{CP}$, with males being more affected than females (Johnston and Hagberg, 2007). Additionally, potential sex differences have been shown in IUGR and following neonatal oxygen exposure. In IUGR, clinical and animal studies have shown contrasting results, some demonstrating male sex as a risk factor (Parkinson et al., 1981; Fung et al., 2012) and others finding no difference or female sex as a risk factor (Reid et al., 2012; Streimish et al., 2012) for poor neurodevelopmental outcomes. In our study, we found no statistical difference between sexes in myelin gene expression in IUGR. Evaluation of sex-differences following hyperoxia exposure in the literature is more limited. In one study, the cellular functions related to energy metabolism, stress, response, and maturation because of oxidative stress were shown to be more pronounced in male versus female-derived OPCs that were exposed to high oxygen for $24 \mathrm{~h}$ (Sunny et al., 2020). In contrast, we demonstrate downregulation of myelin genes in females compared with males following hyperoxia exposure. One potential explanation is that, in our study, our animals were exposed to oxygen over a longer time period of $14 \mathrm{~d}$. The combination of IUGR with hyperoxia resulted in significant downregulation of myelin genes in males compared with females. This is consistent with the male sex being a risk factor for worse neurodevelopment outcomes in other types of perinatal brain injury.

Overall, these findings highlight the complex nature of perinatal brain injury. They also underscore the detrimental effect that oxygen exposure can have on the developing white matter of IUGR infants. Hyperoxia is well known to be implicated in the pathogenesis of bronchopulmonary dysplasia and retinopathy of prematurity (Saugstad, 2001; Weinberger et al., 2002). Our study now adds to the increasing evidence that hyperoxia negatively influences brain maturation and development and results in WMI (Felderhoff-Mueser et al., 2004, 2005; Gerstner et al., 2008; Sunny et al., 2020). Additionally, our study importantly, indicates specific gene networks that contribute to previously demonstrated findings of abnormal myelin formation and motor dysfunction in IUGR and hyperoxia (Chang et al., 2018). The identification of differential gene expression leading to multiple dysregulated signaling pathways known to be integral to CNS myelination, including the Wnt/ $\beta$-catenin and MAPK/Erk pathways in IUGR/hyperoxia, lend insight into how multiple perinatal exposures result in WMI. These findings further stress the need to temper use of therapeutic oxygen in growth restricted infants to minimize WMI.

\section{References}

Abbassi-Daloii T, Kan HE, Raz V, t Hoen PAC (2020) Recommendations for the analysis of gene expression data to identify intrinsic differences between similar tissues. Genomics 112:3157-3165.

Ahlin K, Himmelmann K, Hagberg G, Kacerovsky M, Cobo T, Wennerholm UB, Jacobsson B (2013) Non-infectious risk factors for different types of cerebral palsy in term-born babies: a population-based, case-control study. BJOG 120:724-731.

Anders S, Pyl P, Huber W (2014) HTSeq - A Python framework to work with high-throughput sequencing data. Bioinformatics 31:166-169.

Aslam M, Baveja R, Liang OD, Fernandez-Gonzalez A, Lee C, Mitsialis SA, Kourembanas S (2009) Bone marrow stromal cells attenuate lung injury in a murine model of neonatal chronic lung disease. Am J Respir Crit Care Med 180:1122-1130.

Back SA (2015) Brain injury in the preterm infant: new horizons for pathogenesis and prevention. Pediatr Neurol 53:185-192.

Back SA (2017) White matter injury in the preterm infant: pathology and mechanisms. Acta Neuropathol 134:331-349.

Basilious A, Yager J, Fehlings MG (2015) Neurological outcomes of animal models of uterine artery ligation and relevance to human intrauterine growth restriction: a systematic review. Dev Med Child Neurol 57:420-430.

Battaglia FC, Lubchenco LO (1967) A practical classification of newborn infants by weight and gestational age. J Pediatr 71:159-163.

Bercury KK, Dai J, Sachs HH, Ahrendsen JT, Wood TL, Macklin WB (2014) Conditional ablation of raptor or rictor has differential impact on oligodendrocyte differentiation and CNS myelination. $J$ Neurosci 34:4466-4480.

Birch D, Britt BC, Dukes SC, Kessler JA, Dizon ML (2014) MicroRNAs participate in the murine oligodendroglial response to perinatal hypoxia-ischemia. Pediatr Res 76:334-340.

Blair EM, Nelson KB (2015) Fetal growth restriction and risk of cerebral palsy in singletons born after at least 35 weeks' gestation. Am J Obstet Gynecol 212:520.e1-7.

Brill C, Scheuer T, Bührer C, Endesfelder S, Schmitz T (2017) Oxygen impairs oligodendroglial development via oxidative stress and reduced expression of HIF-1alpha. Sci Rep 7:43000.

Cantone M, Küspert M, Reiprich S, Lai X, Eberhardt M, Göttle P, Beyer F, Azim K, Küry P, Wegner M, Vera J (2019) A gene regulatory architecture that controls region-independent dynamics of oligodendrocyte differentiation. Glia 67:825-843.

Chang JL, Bashir M, Santiago C, Farrow K, Fung C, Brown AS, Dettman RW, Dizon MLV (2018) Intrauterine growth restriction and hyperoxia as a cause of white matter injury. Dev Neurosci 40:344357.

Chapman SD, Farina L, Kronforst K, Dizon M (2018) MicroRNA profile differences in neonates at risk for cerebral palsy. Phys Med Rehabil Int 5:1148.

Chen A, Xiong LJ, Tong Y, Mao M (2013) The neuroprotective roles of BDNF in hypoxic ischemic brain injury. Biomed Rep 1:167-176.

Dahlseng MO, Andersen GL, Irgens LM, Skranes J, Vik T (2014) Risk of cerebral palsy in term-born singletons according to growth status at birth. Dev Med Child Neurol 56:53-58.

Djalali S, Höltje M, Grosse G, Rothe T, Stroh T, Grosse J, Deng DR, Hellweg R, Grantyn R, Hörtnagl H, Ahnert-Hilger G (2005) Effects of brain-derived neurotrophic factor (BDNF) on glial cells and serotonergic neurones during development. J Neurochem 92:616-627.

Dobin A, Davis C, Schlesinger F, Drenkow J, Zaleski C, Jha S, Batut P, Chaisson M, Gingeras T (2013) STAR: ultrafast universal RNAseq aligner. Bioinformatics 29:15-21.

Eriksson L, Haglund B, Odlind V, Altman M, Kieler H (2014) Prenatal inflammatory risk factors for development of bronchopulmonary dysplasia. Pediatr Pulmonol 49:665-672.

Eskenazi B, Fenster L, Sidney S, Elkin E (1993) Fetal growth retardation in infants of multiparous and nulliparous women with preeclampsia. Am J Obstet Gynecol 169:1112-1118.

Ewels P, Magnusson M, Lundin S, Käller M (2016) MultiQC: summarize analysis results for multiple tools and samples in a single report. Bioinformatics 32:3047-3048.

Fancy SP, Baranzini SE, Zhao C, Yuk DI, Irvine KA, Kaing S, Sanai N, Franklin RJ, Rowitch DH (2009) Dysregulation of the Wnt pathway inhibits timely myelination and remyelination in the mammalian CNS. Genes Dev 23:1571-1585.

Fejes Z, Erdei J, Pócsi M, Takai J, Jeney V, Nagy A, Varga A, Bácsi A, Bognár L, Novák L, Kappelmayer J, Nagy B Jr (2020) Elevated proinflammatory cell-free microRNA levels in cerebrospinal fluid of premature infants after intraventricular hemorrhage. Int $\mathrm{J} \mathrm{Mol} \mathrm{Sci}$ 21:6870.

Felderhoff-Mueser U, Bittigau P, Sifringer M, Jarosz B, Korobowicz E, Mahler L, Piening T, Moysich A, Grune T, Thor F, Heumann R, Bührer C, Ikonomidou C (2004) Oxygen causes cell death in the developing brain. Neurobiol Dis 17:273-282. 
Felderhoff-Mueser U, Sifringer M, Polley O, Dzietko M, Leineweber B, Mahler L, Baier M, Bittigau P, Obladen M, Ikonomidou C, Bührer C (2005) Caspase-1-processed interleukins in hyperoxiainduced cell death in the developing brain. Ann Neurol 57:50-59.

Fletcher JL, Murray SS, Xiao J (2018) Brain-derived neurotrophic factor in central nervous system myelination: a new mechanism to promote myelin plasticity and repair. Int J Mol Sci 19:4131.

Freire G, Shevell M, Oskoui M (2015) Cerebral palsy: phenotypes and risk factors in term singletons born small for gestational age. Eur J Paediatr Neurol 19:218-225.

Fu H, Kesari S, Cai J (2012) Tcf7l2 is tightly controlled during myelin formation. Cell Mol Neurobiol 32:345-352.

Fung C, Brown A, Cox J, Callaway C, McKnight R, Lane R (2011) Novel thromboxane A2 analog-induced IUGR mouse model. J Dev Orig Health Dis 2:291-301.

Fung C, Ke X, Brown AS, Yu X, McKnight RA, Lane RH (2012) Uteroplacental insufficiency alters rat hippocampal cellular phenotype in conjunction with ErbB receptor expression. Pediatr Res 72:2-9.

Gaesser JM, Fyffe-Maricich SL (2016) Intracellular signaling pathway regulation of myelination and remyelination in the CNS. Exp Neurol 283:501-511.

Gerstner B, Bührer C, Rheinländer C, Polley O, Schüller A, Berns M, Obladen M, Felderhoff-Mueser U (2006) Maturation-dependent oligodendrocyte apoptosis caused by hyperoxia. J Neurosci Res 84:306-315.

Gerstner B, DeSilva TM, Genz K, Armstrong A, Brehmer F, Neve RL, Felderhoff-Mueser U, Volpe JJ, Rosenberg PA (2008) Hyperoxia causes maturation-dependent cell death in the developing white matter. J Neurosci 28:1236-1245.

Gibbins KJ, Gibson-Corley KN, Brown AS, Wieben M, Law RC, Fung CM (2018) Effects of excess thromboxane A2 on placental development and nutrient transporters in a Mus musculus model of fetal growth restriction. Biol Reprod 98:695-704.

Gonsalvez D, Ferner AH, Peckham H, Murray SS, Xiao J (2016) The roles of extracellular related-kinases 1 and 2 signaling in CNS myelination. Neuropharmacology 110:586-593.

Guedj F, Pennings JL, Ferres MA, Graham LC, Wick HC, Miczek KA, Slonim DK, Bianchi DW (2015) The fetal brain transcriptome and neonatal behavioral phenotype in the Ts1Cje mouse model of Down syndrome. Am J Med Genet A 167A:1993-2008.

Hammond E, Lang J, Maeda Y, Pleasure D, Angus-Hill M, Xu J, Horiuchi M, Deng W, Guo F (2015) The Wnt effector transcription factor 7 -like 2 positively regulates oligodendrocyte differentiation in a manner independent of $\mathrm{Wnt} / \beta$-catenin signaling. J Neurosci 35:5007-5022.

Han BH, Holtzman DM (2000) BDNF protects the neonatal brain from hypoxic-ischemic injury in vivo via the ERK pathway. J Neurosci 20:5775-5781.

Hayakawa M, Takemoto K, Nakayama A, Saito A, Sato Y, Hasegawa M, leda K, Mimura S (2006) An animal model of intrauterine growth retardation induced by synthetic thromboxane a(2). J Soc Gynecol Investig 13:566-572.

Hedtjarn M, Mallard C, Eklind S, Gustafson-Brywe K, Hagberg H (2004) Global gene expression in the immature brain after hypoxiaischemia. J Cereb Blood Flow Metab 24:1317-1332.

Hintz S, Kendrick D, Vohr B, Kenneth Poole W, Higgins R; Nichd Neonatal Research Network (2006) Gender differences in neurodevelopmental outcomes among extremely preterm, extremely-lowbirthweight infants. Acta Paediatr 95:1239-1248.

Hoffmann SA, Hos D, Küspert M, Lang RA, Lovell-Badge R, Wegner M, Reiprich S (2014) Stem cell factor Sox2 and its close relative Sox3 have differentiation functions in oligodendrocytes. Development 141:39-50.

Ishii A, Fyffe-Maricich SL, Furusho M, Miller RH, Bansal R (2012) ERK1/ERK2 MAPK signaling is required to increase myelin thickness independent of oligodendrocyte differentiation and initiation of myelination. J Neurosci 32:8855-8864.

Ishii A, Furusho M, Macklin W, Bansal R (2019) Independent and cooperative roles of the Mek/ERK1/2-MAPK and PI3K/Akt/mTOR pathways during developmental myelination and in adulthood. Glia 67:1277-1295.

Janova H, Böttcher C, Holtman IR, Regen T, van Rossum D, Götz A, Ernst AS, Fritsche C, Gertig U, Saiepour N, Gronke K, Wrzos C, Ribes S, Rolfes S, Weinstein J, Ehrenreich H, Pukrop T, Kopatz J, Stadelmann C, Salinas-Riester G, et al. (2016) CD14 is a key organizer of microglial responses to CNS infection and injury. Glia 64:635-649.

Jochems SP, Marcon F, Carniel BF, Holloway M, Mitsi E, Smith E, Gritzfeld JF, Solórzano C, Reiné J, Pojar S, Nikolaou E, German EL, Hyder-Wright A, Hill H, Hales C, de Steenhuijsen Piters WAA, Bogaert D, Adler H, Zaidi S, Connor V, et al. (2018) Inflammation induced by influenza virus impairs human innate immune control of pneumococcus. Nat Immunol 19:1299-1308.

Johnston MV, Hagberg H (2007) Sex and the pathogenesis of cerebral palsy. Dev Med Child Neurol 49:74-78.

Krämer A, Green J, Pollard J Jr, Tugendreich S (2014) Causal analysis approaches in ingenuity pathway analysis. Bioinformatics 30:523-530.

Lebrun-Julien F, Bachmann L, Norrmén C, Trötzmüller M, Köfeler H, Rüegg MA, Hall MN, Suter U (2014) Balanced mTORC1 activity in oligodendrocytes is required for accurate CNS myelination. J Neurosci 34:8432-8448.

Lee KJ, Berkelhamer SK, Kim GA, Taylor JM, O'Shea KM, Steinhorn $\mathrm{RH}$, Farrow KN (2014) Disrupted pulmonary artery cyclic guanosine monophosphate signaling in mice with hyperoxia-induced pulmonary hypertension. Am J Respir Cell Mol Biol 50:369-378.

Lin HP, Oksuz I, Hurley E, Wrabetz L, Awatramani R (2015) Microprocessor complex subunit DiGeorge syndrome critical region gene 8 (Dgcr8) is required for Schwann cell myelination and myelin maintenance. J Biol Chem 290:24294-24307.

Livak KJ, Schmittgen TD (2001) Analysis of relative gene expression data using real-time quantitative PCR and the 2(-Delta Delta $\mathrm{C}(\mathrm{T})$ ) method. Methods 25:402-408.

Love M, Huber W, Anders S (2014) Moderated estimation of fold change and dispersion for RNA-Seq data with DESeq2. Genome Biol 15:550.

Lürbke A, Hagemeier K, Cui QL, Metz I, Brück W, Antel J, Kuhlmann T (2013) Limited TCF7L2 expression in MS lesions. PLoS One 8: e72822.

Mandruzzato G, Antsaklis A, Botet F, Chervenak FA, Figueras F, Grunebaum A, Puerto B, Skupski D, Stanojevic M; WAPM (2008) Intrauterine restriction (IUGR). J Perinat Med 36:277-281.

McAdam BF, Byrne D, Morrow JD, Oates JA (2005) Contribution of cyclooxygenase-2 to elevated biosynthesis of thromboxane A2 and prostacyclin in cigarette smokers. Circulation 112:1024-1029.

Mestan KK, Check J, Minturn L, Yallapragada S, Farrow KN, Liu X, Su E, Porta N, Gotteiner N, Ernst LM (2014) Placental pathologic changes of maternal vascular underperfusion in bronchopulmonary dysplasia and pulmonary hypertension. Placenta 35:570574.

Mirza MA, Ritzel R, Xu Y, McCullough LD, Liu F (2015) Sexually dimorphic outcomes and inflammatory responses in hypoxic-ischemic encephalopathy. J Neuroinflammation 12:32.

Mor O, Stavsky M, Yitshak-Sade M, Mastrolia SA, Beer-Weisel R, Rafaeli-Yehudai T, Besser L, Hamou B, Mazor M, Erez O (2016) Early onset preeclampsia and cerebral palsy: a double hit model? Am J Obstet Gynecol 214:105.e1-9.

Narang R, Carter K, Muncie C, Pang Y, Fan LW, Feng Y, Ojeda NB, Bhatt AJ (2019) Intrauterine growth restriction and neonatal hypoxic ischemic brain injury causes sex-specific long-term neurobehavioral abnormalities in rats. J Neurosci Res 97:661-672.

Narayanan SP, Flores Al, Wang F, Macklin WB (2009) Akt signals through the mammalian target of rapamycin pathway to regulate CNS myelination. J Neurosci 29:6860-6870.

Olivier P, Baud O, Bouslama M, Evrard P, Gressens P, Verney C (2007) Moderate growth restriction: deleterious and protective effects on white matter damage. Neurobiol Dis 26:253-263. 
Parkinson CE, Wallis S, Harvey D (1981) School achievement and behaviour of children who were small-for-dates at birth. Dev Med Child Neurol 23:41-50.

Ponnusamy V, Kapellou O, Yip E, Evanson J, Wong LF, MichaelTitus A, Yip PK, Shah DK (2016) A study of microRNAs from dried blood spots in newborns after perinatal asphyxia: a simple and feasible biosampling method. Pediatr Res 79:799-805.

Ramani M, van Groen T, Kadish I, Bulger A, Ambalavanan N (2013) Neurodevelopmental impairment following neonatal hyperoxia in the mouse. Neurobiol Dis 50:69-75.

Reid MV, Murray KA, Marsh ED, Golden JA, Simmons RA, Grinspan JB (2012) Delayed myelination in an intrauterine growth retardation model is mediated by oxidative stress upregulating bone morphogenetic protein 4. J Neuropathol Exp Neurol 71:640-653.

Rideau Batista Novais A, Pham H, Van de Looij Y, Bernal M, Mairesse J, Zana-Taieb E, Colella M, Jarreau PH, Pansiot J, Dumont F, Sizonenko S, Gressens P, Charriaut-Marlangue C, Tanter M, Demene C, Vaiman D, Baud O (2016) Transcriptomic regulations in oligodendroglial and microglial cells related to brain damage following fetal growth restriction. Glia 64:2306-2320.

Ritter J, Schmitz T, Chew LJ, Bührer C, Möbius W, Zonouzi M, Gallo V (2013) Neonatal hyperoxia exposure disrupts axon-oligodendrocyte integrity in the subcortical white matter. J Neurosci 33:89909002.

Rozance PJ, Seedorf GJ, Brown A, Roe G, O'Meara MC, Gien J, Tang JR, Abman SH (2011) Intrauterine growth restriction decreases pulmonary alveolar and vessel growth and causes pulmonary artery endothelial cell dysfunction in vitro in fetal sheep. Am J Physiol Lung Cell Mol Physiol 301:L860-871.

Russo PST, Ferreira GR, Cardozo LE, Bürger MC, Arias-Carrasco R, Maruyama SR, Hirata TDC, Lima DS, Passos FM, Fukutani KF, Lever M, Silva JS, Maracaja-Coutinho V, Nakaya HI (2018) CEMiTool: a bioconductor package for performing comprehensive modular co-expression analyses. BMC Bioinformatics 19:56.

Saugstad OD (2001) Chronic lung disease: oxygen dogma revisited. Acta Paediatr 90:113-115.

Schmitz T, Ritter J, Mueller S, Felderhoff-Mueser U, Chew LJ, Gallo V (2011) Cellular changes underlying hyperoxia-induced delay of white matter development. J Neurosci 31:4327-4344.

Schroder HJ (2003) Models of fetal growth restriction. Eur J Obstet Gynecol Reprod Biol 110 [Suppl 1]:S29-S39.

Silbereis JC, Huang EJ, Back SA, Rowitch DH (2010) Towards improved animal models of neonatal white matter injury associated with cerebral palsy. Dis Model Mech 3:678-688.

Spaull R, McPherson B, Gialeli A, Clayton A, Uney J, Heep A, Cordero-Llana Ó (2019) Exosomes populate the cerebrospinal fluid of preterm infants with post-haemorrhagic hydrocephalus. Int J Dev Neurosci 73:59-65.

Streimish IG, Ehrenkranz RA, Allred EN, O'Shea TM, Kuban KC, Paneth N, Leviton A; ELGAN Study Investigators (2012) Birth weight- and fetal weight-growth restriction: impact on neurodevelopment. Early Hum Dev 88:765-771.

Streja E, Miller JE, Wu C, Bech BH, Pedersen LH, Schendel DE, Uldall P, Olsen J (2015) Disproportionate fetal growth and the risk for congenital cerebral palsy in singleton births. PLoS One 10: e0126743.
Sunny DE, Hammer E, Strempel S, Joseph C, Manchanda H, Ittermann T, Hübner S, Weiss FU, Völker U, Heckmann M (2020) Nup133 and ER $\alpha$ mediate the differential effects of hyperoxia-induced damage in male and female OPCs. Mol Cell Pediatr 7:10.

Tolcos M, Bateman E, O'Dowd R, Markwick R, Vrijsen K, Rehn A, Rees $S$ (2011) Intrauterine growth restriction affects the maturation of myelin. Exp Neurol 232:53-65.

Tolcos M, Petratos S, Hirst JJ, Wong F, Spencer SJ, Azhan A, Emery B, Walker DW (2017) Blocked, delayed, or obstructed: what causes poor white matter development in intrauterine growth restricted infants? Prog Neurobiol 154:62-77.

Valerio-Gomes B, Guimaraes DM, Szczupak D, Lent R (2018) The absolute number of oligodendrocytes in the adult mouse brain. Front Neuroanat 12:90.

van Dam S, Vosa U, van der Graaf A, Franke L, de Magalhaes JP (2018) Gene co-expression analysis for functional classification and gene-disease predictions. Brief Bioinform 19:575-592.

van Tilborg E, de Theije CGM, van Hal M, Wagenaar N, de Vries LS, Benders MJ, Rowitch DH, Nijboer CH (2018) Origin and dynamics of oligodendrocytes in the developing brain: implications for perinatal white matter injury. Glia 66:221-238.

Voineagu I, Wang X, Johnston P, Lowe JK, Tian Y, Horvath S, Mill J, Cantor RM, Blencowe BJ, Geschwind DH (2011) Transcriptomic analysis of autistic brain reveals convergent molecular pathology. Nature 474:380-384.

Volpe JJ, Kinney HC, Jensen FE, Rosenberg PA (2011) The developing oligodendrocyte: key cellular target in brain injury in the premature infant. Int J Dev Neurosci 29:423-440.

Vondran MW, Clinton-Luke P, Honeywell JZ, Dreyfus CF (2010) $\mathrm{BDNF}+/$ - mice exhibit deficits in oligodendrocyte lineage cells of the basal forebrain. Glia 58:848-856.

Wahl SE, McLane LE, Bercury KK, Macklin WB, Wood TL (2014) Mammalian target of rapamycin promotes oligodendrocyte differentiation, initiation and extent of CNS myelination. J Neurosci 34:4453-4465.

Wang Z, Liu Y, Shao M, Wang D, Zhang Y (2018) Combined prediction of miR-210 and miR-374a for severity and prognosis of hypoxic-ischemic encephalopathy. Brain Behav 8:e00835.

Weinberger B, Laskin DL, Heck DE, Laskin JD (2002) Oxygen toxicity in premature infants. Toxicol Appl Pharmacol 181:60-67.

Zhang G, Ye M, Li M (2020) Deregulated miR-384 serves as a biomarker in neonatal hypoxic-ischemic encephalopathy and alleviates microglia-mediated neuroinflammation. Mol Biol Rep 47:5411-5420.

Zhang Y, Chen K, Sloan SA, Bennett ML, Scholze AR, O'Keeffe S, Phatnani HP, Guarnieri P, Caneda C, Ruderisch N, Deng S, Liddelow SA, Zhang C, Daneman R, Maniatis T, Barres BA, Wu JQ (2014) An RNA-sequencing transcriptome and splicing database of glia, neurons, and vascular cells of the cerebral cortex. J Neurosci 34:11929-11947.

Zhao C, Ma D, Zawadzka M, Fancy SP, Elis-Williams L, Bouvier G, Stockley JH, de Castro GM, Wang B, Jacobs S, Casaccia P, Franklin RJ (2015) Sox2 sustains recruitment of oligodendrocyte progenitor cells following CNS demyelination and primes them for differentiation during remyelination. J Neurosci 35:11482-11499. 